\author{
Journal of Research of the National Bureau of Standards \\ Volume 90, Number 2, March-April 1985
}

\title{
Standards for Measurement of the Critical Fields of Superconductors
}

\author{
F. R. Fickett \\ National Bureau of Standards, Boulder, CO 80303
}

\begin{abstract}
Accepted: November 21, 1984
The origins, definitions, and measurement of the various critical magnetic fields associated with superconductors are reviewed. The potential need for a consensus standard for the measurement of these fields is evaluated. Measurement techniques as practiced both in industry and in the national laboratories and extrapolation techniques commonly used to determine the upper critical fields of the newer materials are presented. Sources of error in the experimental determination of critical fields are assessed for the various common techniques. A comprehensive bibliography of the modern literature on critical field measurement and interpretation is included.
\end{abstract}

Key words: critical field; critical parameters; magnetic field; standards; superconductors.

\section{Introduction}

The literature of the field of superconductivity is replete with numerous critical magnetic fields. Several $\left(H_{\mathrm{c}}, H_{\mathrm{cl}}, H_{\mathrm{c} 2}\right)$ have universal application and acceptance, while many others are defined for subtle and specific purposes [e.g., $B_{\mathrm{c} 2 \mathrm{~m}}^{*}(0)$ ]. Our goal is to evaluate whether there is a need for a measurement standard for critical field similar to one developed recently for critical current [1]. ${ }^{1}$ We consider only superconductive materials that are either commercial items now or those that have the potential of being so in the foreseeable future. This criterion might seem to allow us to ignore many of the more difficult aspects of the problem, such as the effect of surface superconductivity. Yet, our survey of the literature indicates that frequently these effects do appear in rather mundane measurements and can alter the results significantly. Thus, we have included most of the effects that have been observed to date during attempts

About the Author, Paper: F. R. Fickett is a physicist with the NBS Electromagnetic Technology Division in Boulder, CO. His work was supported by the Department of Energy through the Office of Fusion Energy and the Division of High Energy Physics.

${ }^{1}$ Numbers in brackets refer to literature references. to determine the critical fields. However, for practical purposes, effects that take place below a critical current value of about $10^{4} \mathrm{~A} / \mathrm{cm}^{2}$ are seldom of interest, which removes us from the more esoteric regions of the $\mathrm{J}-\mathrm{H}$ and $\mathrm{T}-\mathrm{H}$ curves. There are, of course, exceptions such as pinning studies. Similarly, the more exotic materials, such as the Chevrel-phase conductors, are not of immediate practical importance. However, that situation could easily change. Thus, although our concentration is on the conductors that are commercially available, these new materials with great potential are also considered.

The terminology used here for the general references to the field of superconductivity is that presented in the several review documents on terminology prepared by our group [2,3] and in the new ASTM standard for superconductor terminology [4]. Definitions of the various critical fields are given both in the discussion of the theory and of the extrapolation techniques.

In the recent past, the differentiation of $B$ and $H$ was a matter left to those who worried about the basic physics of magnetism. Use of the cgs system was entirely acceptable and magnetism and superconductivity did not coexist. Under these conditions, the gauss and the oersted were effectively equivalent and, in fact, were often used interchangeably. At present, however, SI units are rapidly gaining acceptance and more is being written regarding the relationships of magnetism and 
superconductivity [5]. Both situreions require that we use more care in the application of these terms. The reader interested in the basics of magnetism is referred to our recent publication on magnetic effects at low temperatures [6]. Table 1 lists definitions and unit con- versions for mag-1etic quantities.

The determining equation (in SI) is:

$$
B=\mu_{0}(H+M) \text {, }
$$

where $B$ is the magnetic flux density (or magnetic in-

Table 1. Units for magnetic properties.

\begin{tabular}{|c|c|c|c|c|}
\hline Property & Symbol & Gaussian \& $\mathrm{cgs}$ emu ${ }^{\mathrm{a}}$ & $\begin{array}{l}\text { Conversion } \\
\text { factor, } C^{b}\end{array}$ & SI \& rationalized $\mathrm{mks}^{\mathrm{c}}$ \\
\hline $\begin{array}{l}\text { Magnetic flux density, } \\
\text { magnetic induction }\end{array}$ & $B$ & gauss $(\mathrm{G})^{\mathrm{c}}$ & $10^{-4}$ & tesla $(\mathrm{T}), \mathrm{Wb} / \mathrm{m}^{2}$ \\
\hline Magretic fux & $\delta$ & maxwell $(M x) G\left(\mathrm{~cm}^{2}\right.$ & $10^{-8}$ & weber (Whi, volt sacond (V.s) \\
\hline $\begin{array}{l}\text { Magnetic field strength, } \\
\text { magnetizing force }\end{array}$ & $H$ & oersted $(\mathrm{Oe})^{\mathrm{e}}$ & $10^{3} / 4 \pi$ & ampere per meter $(\mathrm{A} / \mathrm{m})^{\mathrm{r}}$ \\
\hline (Volume) magnetizations & $M$ & $\mathrm{emu} / \mathrm{cm}^{3 \mathrm{~h}}$ & $10^{3}$ & $\mathrm{~A} / \mathrm{m}$ \\
\hline (Volume) magnetization & $4 \pi M$ & $G$ & $10^{3} / 4 \pi$ & $\mathrm{A} / \mathrm{m}$ \\
\hline $\begin{array}{l}\text { Magnetic polarization, } \\
\text { intensity of magnetization }\end{array}$ & $J, 1, M$ & $\mathrm{emu} / \mathrm{cm}^{3}$ & $4 \pi \times 10^{-4}$ & $\mathrm{~T}, \mathrm{~W} b / \mathrm{m}^{2 i}$ \\
\hline (Mass) magnetization & $\sigma, M$ & $\mathrm{emu} / \mathrm{g}$ & $\begin{array}{l}1 \\
4 \pi \times 10^{-7}\end{array}$ & $\begin{array}{l}\mathrm{A} \cdot \mathrm{m}^{2} / \mathrm{kg} \\
\mathrm{Wb} \cdot \mathrm{m} / \mathrm{kg}\end{array}$ \\
\hline (Volume) suscept:bi.ity & $x, r$ & dimersionless, em. $\lambda^{\prime} \mathrm{cm}^{3}$ & $\begin{array}{l}4 \pi \\
(4 \pi)^{2} \times 10^{-i}\end{array}$ & $\begin{array}{l}\text { dimens or.less } \\
\text { henry per meter }(\mathbf{H} / \mathrm{m}), \mathrm{Wb} /(\mathrm{A} \cdot \mathrm{m})\end{array}$ \\
\hline (Mass) susceptibility & $x_{\rho}, k_{p}$ & $\mathrm{~cm}^{3} / \mathrm{g}$, emu/g & $\begin{array}{l}4 \pi \times 10^{-3} \\
(4 \pi)^{2} \times 10^{-10}\end{array}$ & $\begin{array}{l}\mathrm{m}^{3} / \mathrm{kg} \\
\mathrm{H} \cdot \mathrm{m}^{2} / \mathrm{kg}\end{array}$ \\
\hline (Molas) susceptibility & $\chi_{\text {mol }}, \kappa_{\text {mos }}$ & $\mathrm{cm}^{3} / \mathrm{mol}_{\mathrm{a}}$ emu/mol & $\begin{array}{l}4 \pi \times 10^{-6} \\
(4 \pi)^{2} \times 10^{-13}\end{array}$ & $\begin{array}{l}\mathrm{m}^{3} / \mathrm{mol} \\
\mathrm{H} \cdot \mathrm{m}^{2} / \mathrm{mol}\end{array}$ \\
\hline Magnetic moment & $m$ & emu, erg $/ G$ & $10^{-3}$ & $A \cdot m^{2}$, joule per tesla $(J / T)$ \\
\hline Magnetis dipole moment & $j$ & emu, erg/G & $4 \pi \times 10^{-10}$ & $w b \cdot m^{i}$ \\
\hline Permeability & $\mu$ & dimensionless & $4 \pi \times 10^{-7}$ & $\mathrm{H} / \mathrm{m}$ \\
\hline Relalive fe-meabil: $1 y^{\mathbf{i}}$ & $\boldsymbol{H}_{\text {r }}$ & not defined & & dinensionless \\
\hline $\begin{array}{l}\text { (Volume) energy density, } \\
\text { energy product }\end{array}$ & $W, C$ & $\mathrm{erg} / \mathrm{cm}^{3}$ & $10^{-:}$ & $J / m^{3}$ \\
\hline Derttagnetization factor & $D . N$ & dimensionless & $1 / 4 \pi$ & dimensionless \\
\hline
\end{tabular}

"Gaussian units and egs emu are the same for magnetic properties. The defining relation is $B=H+4 \pi M$.

'Multiply a number in Gaussian units by $C$ co convert it to $S I$ (e.g., $1 \mathrm{G} \times 10^{-4} \mathrm{~T} / \mathrm{G}=10^{-4} \mathrm{~T}$ ).

'SI (Spsceme Internationat d'Unités' has been adopted by the National Bureau of Standards. Where two conversion factors are given, the upper one is recognized under, or consistent witth, SI and is based on the detinition $B=\mu_{0}(H+M)$, where $\mu_{0}=4 \pi \times 10^{-7} \mathrm{H} / \mathrm{m}$. The lower one is not recognized under SI and is based on the definition $B=\mu_{0} H+J$, where the symbal $I$ ar $M$ is of ten used in place of $J$.

d! gauss $=10^{5}$ gamma $(\gamma)$.

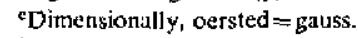

'A/m was often expressed as "ampere-1urn per meter" when used for magnelic field strength.

BMagne; mament par drit velume.

"T he dasigniltior. "ernu" is not a uzu.

Recognized under SII, even though based on the difinttion $B=\mu_{0} H+J$. See footnote $\omega$

$j_{\mu,}=\mu / \mu_{0}=1+\chi_{1}$ all in SI. $\mu$, is equal to Gaussian $\mu$

R. B. Goldfarb and F. R. Fickett, National Bureau of Standards, Boulder, Co 80303 , July 1984 
duction) in teslas, $H$ is the magnetic field strength in amperes/meter, $M$ is the (volume) magnetization in amperes/meter, and $\mu_{0}$ is the permeability of free space $\left(4 \pi \times 10^{-7}\right.$ henrys/meter). The important distinction here is that $H$ is an applied field, $M$ is a property of the material and, thus, $B$ is a mixed quantity. Because of this distinction, we suggest that the proper quantity for the critical fields discussed here is $H$; it is the applied field value at which the transition occurs. This choice leads to the unfortunate side effect which has plagued us for years-few researchers have an innate feel for the units of amperes/meter. This, in turn, leads to the common fix used by many authors of multiplying the number by $\mu_{0}$ and then using the units of teslas, which are more familiar. As an aside: the correct abbreviation for the tesla is $\mathrm{T}$ and the correct plural name is teslas (not capitalized) [7].

Another subject that sometimes leads to confusion in the realm of magnetic measurements is the demagnetization factor, $N$. In magnetizable bodies, the poles that appear under the influence of the applied field give a return field through the body that has the effect of lowering the actual value of $H$ within the material. This is a geometric effect and is discussed in detail in our publication [6]. It is of concern with superconductors only as long as they are in the perfect Meissner state below $H_{\mathrm{cl}}$, a region that is seldom of practical interest. However, in this region the effect is important and must be accounted for either in the data processing or by choice of a sample geometry that minimizes the effect (a long ellipsoid with its long axis parallel to the applied field).

At first glance, the importance of critical field in practical applications is not obvious. Certainly high values are desired for applications in very high field magnets, but only if reasonable critical currents can also be achieved. However, the upper critical field and its behavior as a function of temperature and critical current are topics of major importance to the theoretical understanding of type II superconductivity, which in turn will almost certainly lead to better materials for the future. A prime example of this is the effort now afoot to create a higher critical field in NbTi alloys by third element additions [8]. The work is crucially dependent on accurate determination of the critical fields. Another example is the application of critical field values in the treatment of scaling of strain effects in high field superconductors.

In the remainder of this paper we will discuss how the various critical fields arise in theory and how they are related to other parameters. The techniques used for the determination of the fields are reviewed with discussion of the accuracy, precision, and experimental difficulties involved. Since high critical fields are most often deter- mined by extrapolation rather than direct measurement, some time is spent discussing the various extrapolation schemes and their merits and problems, including controversies that have arisen related to the measurement and interpretation of modern data. The final section presents our conclusions and suggestions for how best to apply the concept of standards to this measurement problem.

\section{Theory and Definitions}

The theoretical background for the various definitions associated with critical magnetic fields is adequately covered in many texts on superconductivity. Cody [9] provides a particularly complete listing of the equations. Here we present just enough theory to allow us to define the terms that are essential to understanding the problems involved with the determination of critical fields. Where we have an option we will choose the simplest workable definition and leave the subtle details to the theorists. All the terms arise in one way or another from the Bardeen-Cooper-Schrieffer (BCS) or Ginzburg-Landau-Abrikosov-Gorkov (GLAG) theories. Application of these concepts to the theory of practical materials, such as the multifilamentary superconductors, is an active area of research at the present time [10-12].

Type I superconductors are included here for completeness, but are nearly totally neglected thereafter, since our concern is with the high-field type II materials.

\subsection{Basic Behavior}

Here we present the basics of type I and type II superconductivity. The complications that arise in the various limits are discussed in section 2.2. To start, we define five parameters:

Penetration depth, $\lambda$. This characteristic length is a measure of the depth of flux penetration into a superconductor. The currents which prevent flux penetration into the interior of the material flow in a layer of this thickness. The exact temperature dependence of this parameter is open to debate, but it decreases monotonically with increasing temperature and rapidly drops to zero near the critical temperature. Typical measured values at $4 \mathrm{~K}$ for $\mathrm{NbTi}$ and $\mathrm{Nb}_{3} \mathrm{Sn}$ are hundreds of nanometers, but with a large spread. For elemental superconductors, $\lambda$ is tens of nanometers.

Coherence length, $\xi$. This length is a measure of the typical size of the Cooper pairs. Looked at differently, it can be taken as the minimum thickness of the interface between superconducting and normal regions. Specifics depend on the particular theoretical treatment. The 
GLAG coherence length is temperature dependent, being inversely proportional to the temperature difference from the critical value. In impure materials, the coherence length value is also influenced by the electron mean free path. Typical values are around $5 \mathrm{~nm}$ for type II superconductors and considerably larger for type I (A1 $\sim 1600 \mathrm{~nm}, \mathrm{~Pb} \sim 83 \mathrm{~nm}$ ).

Ginzburg-Landau parameter, $\kappa$. This is the dimensionless ratio of the two parameters above.

$$
\kappa=\lambda / \xi .
$$

It is roughly temperature independent. $\kappa$ is used as the parameter that distinguishes between type I and type II superconductors; those with a value $<1 / \sqrt{2}$ are type I.

Flux quantum, $\phi_{0}$. The fundamental unit of magnetic flux.

$$
\phi_{0}=h / 2 \mathrm{e}=2.068 \times 10^{-15} \mathrm{~Wb} .
$$

Normal state resistivity, $\rho_{\mathrm{n}}$. The classical electrical resistivity as measured just above the superconducting transition unless stated otherwise.

The general behavior of a superconductor in a field is shown in figure 1. The shape is chosen to minimize demagnetization effects. The conductor is in the Meissner state with $B=0$ in the bulk of the material. The superconductor distorts the field lines in its vicinity.

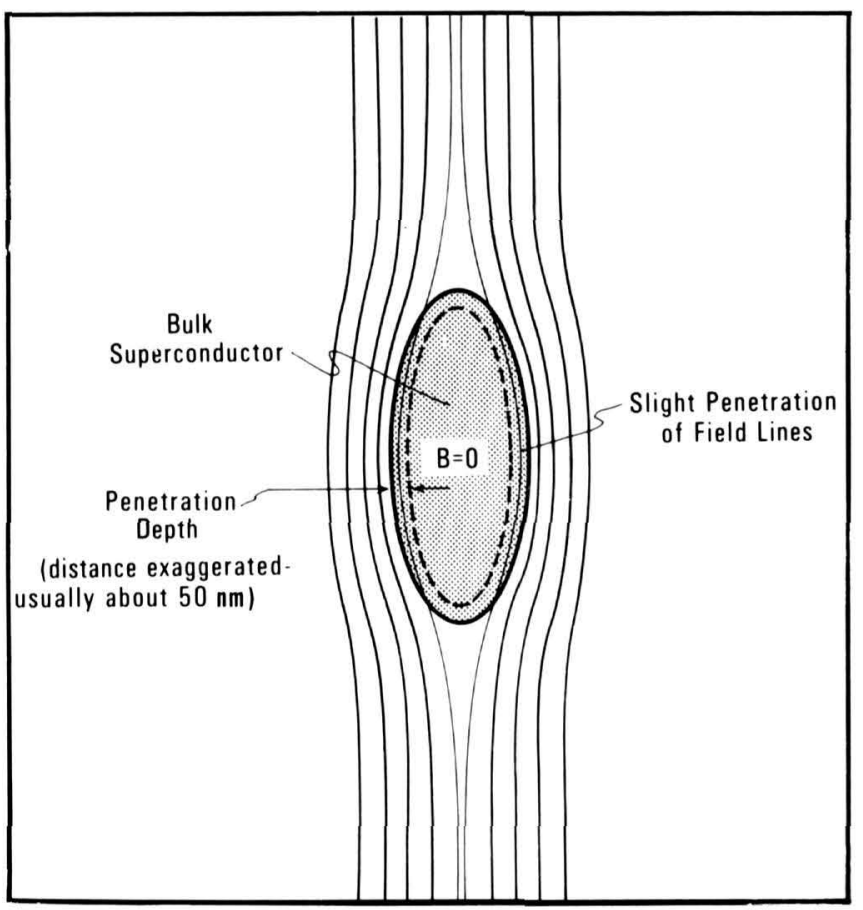

Figure 1-Superconductor behavior in the Meissner state showing the effect of the penetration depth [2].
This picture is valid for either type of superconductor at this point, i.e., below the first critical field value. The behavior of various parameters for each of the two types is shown in figure 2 .

Type I superconductors. These materials are pure metals and the critical field transition is first order. The coherence length is larger than the penetration depth, and a mixed state (see below) is energetically un-
Type I
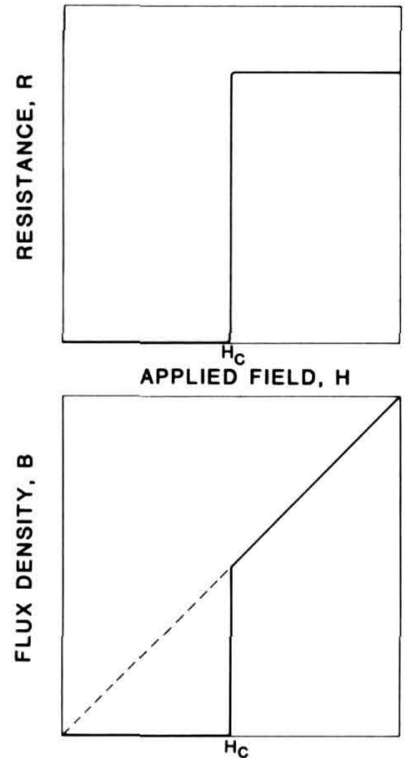

APPLIED FIELD, $\mathrm{H}$
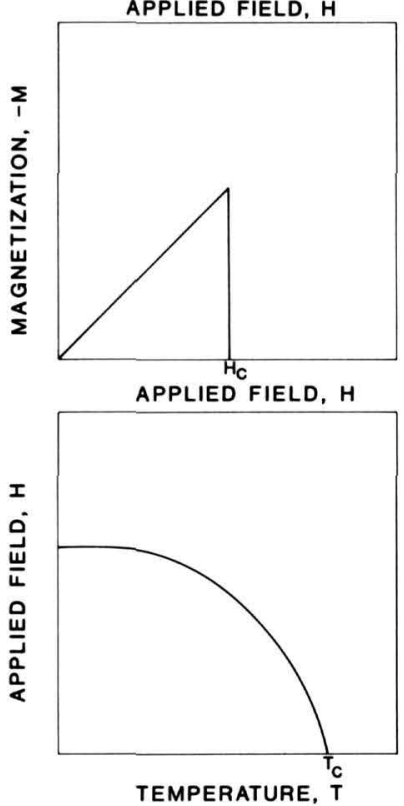

Type II
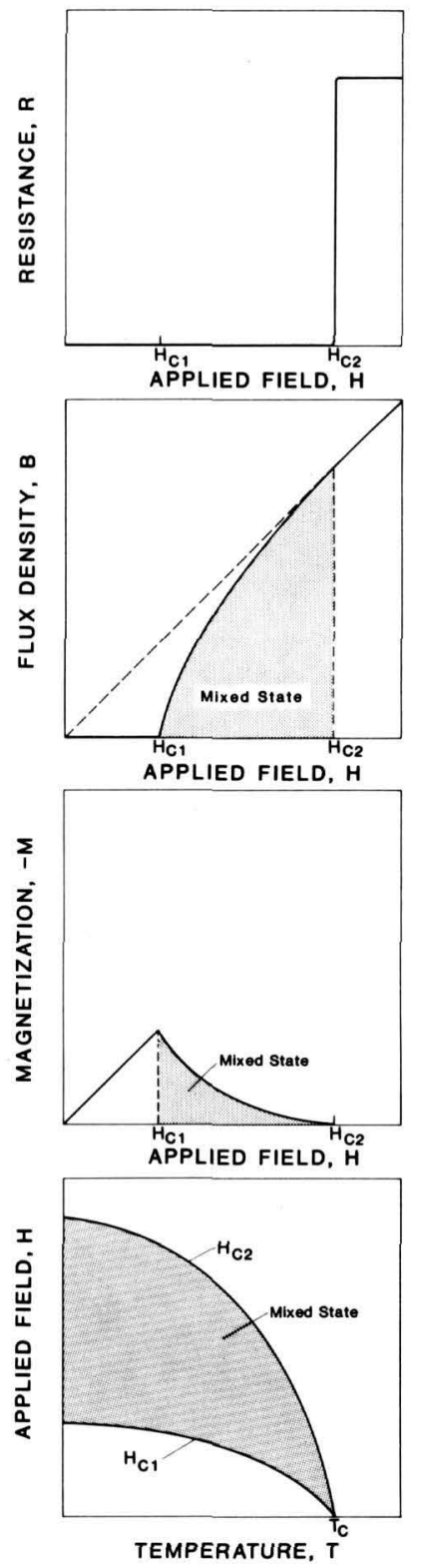

Figure 2-The parameters most often used in the determination of critical field and how they vary with field for both type I and type II superconductors. 
favorable. Thus, at $H_{\mathrm{c}}$ the superconducting state collapses entirely. The critical field is temperature dependent, with an approximately parabolic dependence on $T / T_{\mathrm{c}}$. Below the critical field the flux density is zero in the bulk and the magnetic susceptibility $(M / H)$ is -1 (SI units), i.e., the material is perfectly diamagnetic. The resistive transition is sharp for these materials, sufficiently so that some are used as temperature standards.

Type II superconductors. These are the materials of primary commercial interest. All of the high field superconductors are a subgroup of this type. The materials tend to be alloys and compounds. They also have a region of perfect diamagnetism that extends only up to a rather small lower critical field, $H_{\mathrm{cl}}$, at which a second order transition to the mixed state occurs. The behavior of the related properties is shown on the right side of figure 2. The fact that the penetration depth is greater than the coherence length gives rise to a negative surface energy between the superconducting and normal regions and the mixed state becomes energetically favorable at a relatively low field $\left(H_{\mathrm{it}}\right)$. The structure of this state is as shown in figure 3. It starts when quantized flux bundles, usually of magnitude $\phi_{v}$, penetrate the interior of the material. The flux is concentrated in the normal core which is surrounded by circulating supercurrents and, in the limit of zero applied field, it decays to zero in a distance from the core equal to the penetration depth. The density of superconducting electron pairs, which is zero in the core, reaches its equilibrium value in a distance approximately equal to the coherence

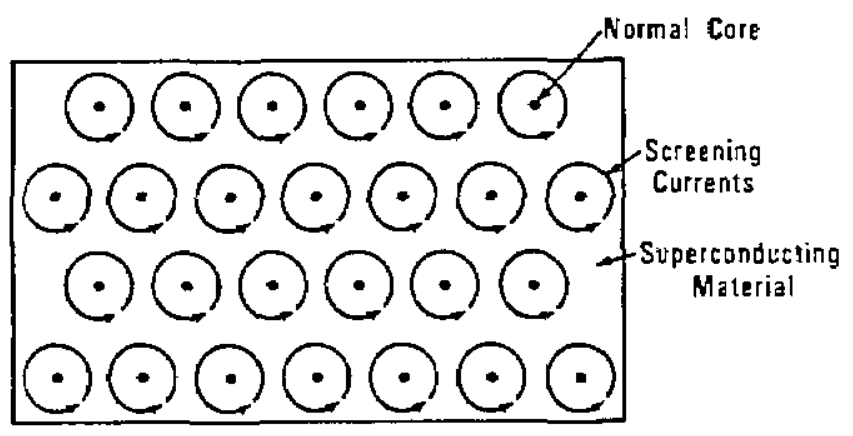

TOP VIEW

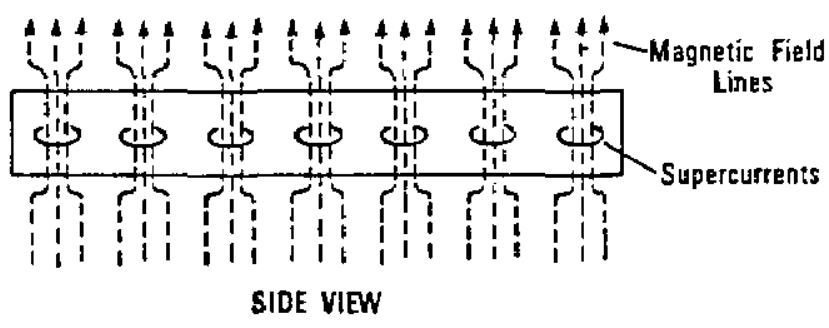

Figure 3-Flux penetration in the mixed state of type II superconductors showing the vortex structure and the flux lattice [13]. length. With increasing applied field, the number of these flux vortices increases and they form a triangular array, the flux lattice, with an equilibrium separation that varies as $B^{-\frac{1}{2}}$. At the upper critical field, $H_{\mathrm{c} 2}$, the flux lattice vanishes and bulk superconductivity is destroyed. The effect of this scenario on the bulk properties can be seen in figure 2 . The resistance (measured with sufficiently low current) does not show the transformation until $H_{\mathrm{c} 2}$ because continuous superconducting paths still exist through the material up to this point. However, the amount of current that can be carried, the critical current density, decreases rapidly as the upper critical field is approached, as shown by the data in figure $4 . H_{\mathrm{c} 2}$ is temperature dependent, and the slope of

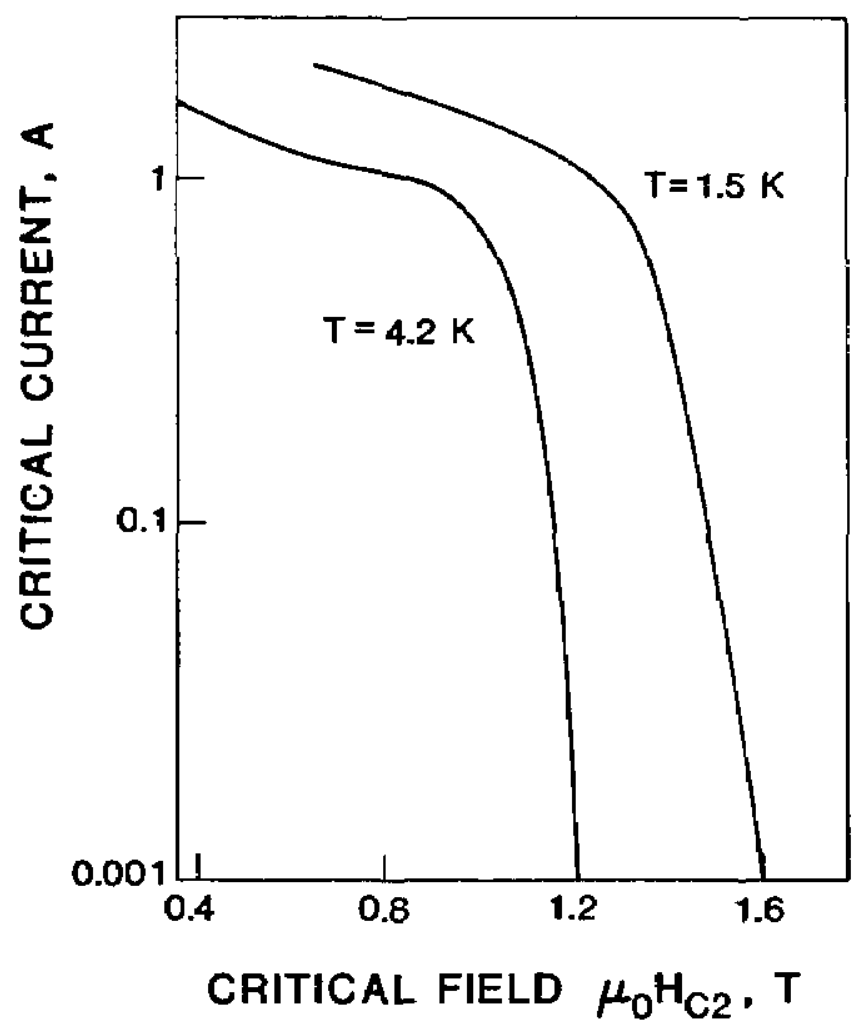

Figure 4-Variation of the critical current near the upper critical field. Data from [14].

the curve near $T_{\mathrm{c}}$ is an important parameter in the detailed theory of these materials. For some materials, very high values of $H_{\mathrm{c} 2}$ may occur as shown in figure 5; it has been said that critical field values on the order of a $100 \mathrm{~T}$ are not ruled out by existing theory [15].

There are two classes of superconductors in this category, intrinsic and impurity dominated. In theory almost any superconductor can be put into the latter category by the introduction of impurities or disorder to raise the normal state resistivity. Hulm and Matthias [12] suggest as an approximate criterion for intrinsic superconductors that the critical temperature should exceed 


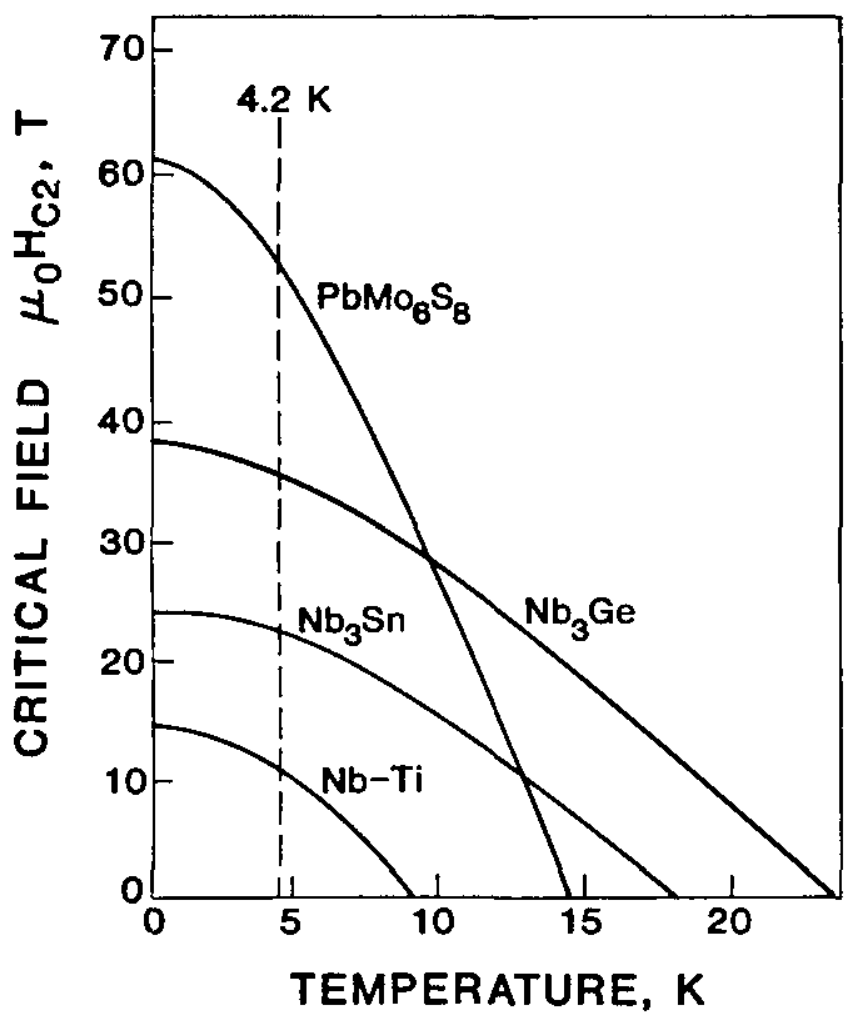

Figure 5-Behavior of critical field with temperature for several modern superçonductors.

$8 \mathrm{~K}$. The relationship between the critical field and critical temperature of the two types is quite different. For the intrinsic materials

$$
H_{\mathrm{c} 2} \propto\left(\gamma T_{\mathrm{c}}\right)^{2}
$$

where $\gamma$ is the electronic specific heat coefficient (proportional to the density of states). The impurity dominated materials give

$$
H_{\mathrm{c} 2} \propto \rho_{\mathrm{n}} \gamma T_{\mathrm{c}} .
$$

Thus it is possible (with some assumptions) to separate the two classes of materials by plotting $H_{\mathrm{c} 2}$ against $\gamma T_{\mathrm{c}}$.

\subsection{Complications}

In addition to the rather straightforward considerations above, the type II materials have other, more complex, aspects to their behavior. The theoretical basis of most of these is presented here, again, in just enough detail to describe the effect. The interested reader should consult the pertinent references. Explanation of these effects requires the introduction of new critical fields. These fields are usually not directly measurable.
Surface critical field, $H_{\mathrm{c} 3}$. Above $H_{\mathrm{c} 2}$ a material may still carry a small supercurrent in a surface layer of thickness approximately equal to the coherence length. This occurs in situations that are usually not encountered in the measurement of practical materials, such as when the field is parallel to a free conductor surface. It is not seen if the field is perpendicular to the sample nor if the sample surface is in intimate contact with normal conductor. The surface current persists with increasing field up to the surface critical field. $H_{\mathrm{c} 3}$ is related to $H_{\mathrm{c} 2}$ by

$$
H_{\mathrm{c} 3}=\alpha H_{\mathrm{c} 2},
$$

where $\alpha$ is about 1.7. Figures 6 and 7 show the effect on the magnetization and the field-temperature plots. If the requisite conditions are met, the effect can cause serious errors in critical field determinations, especially if the measurement is made by a resistive technique.

Magnetic, spin, and scattering effects. Many effects work to alter the simple picture of the origin of the upper critical field presented above. Foremost among these is the paramagnetism of the normal state, but contributions also arise from the paramagnetism of the superconducting state, spin-orbit scattering effects, and electron-phonon coupling. Because of this, the full GLAG theory expression for the upper critical field for high values of $\kappa$,

$$
\mu_{0} H_{\mathrm{c} 2}^{*}(0)=3.11 \times 10^{3} \rho_{\mathrm{n}} \gamma \mathrm{T}_{\mathrm{c}} \text { (teslas), }
$$

defines a critical field that is higher than that usually measured. Normal state paramagnetism causes the

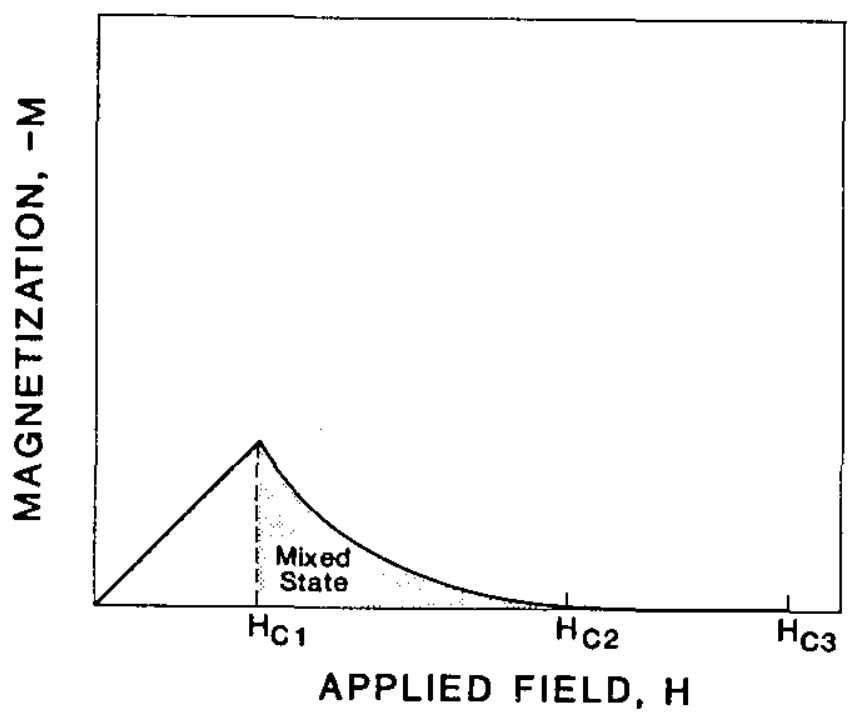

Figure 6-Magnetization curve for a type II material showing the extension to $H_{\mathrm{e} 3}$. 


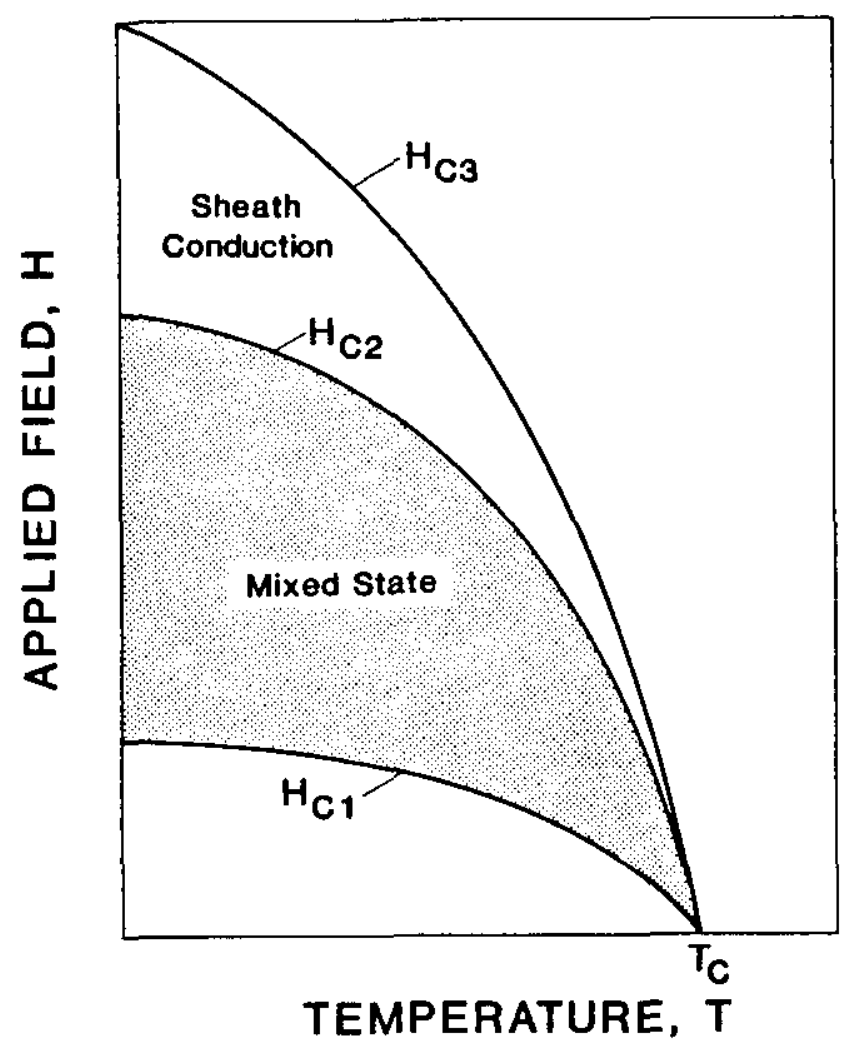

Figure 7-Applied field as a function of temperature for a type II superconductor showing the region of sheath conduction below $H_{\mathrm{c} 3}$.

actual value to be lower. It is possible to calculate this paramagnetically limited transition field with the result,

$$
\mu_{0} H_{\mathrm{p}}(0)=1.84 \mathrm{~T}_{\mathrm{c}} \text { (teslas). }
$$

Another symbol for this field that is sometimes used is $H_{\mathrm{c} 2}^{\mathrm{p}}(0)$. Further modifications to the theory to account for paramagnetic effects lead to another critical field,

$$
H_{\mathrm{c} 2}^{* *}(0)=H_{\mathrm{c} 2}^{*}(0)\left(1+\alpha^{2}\right)^{-1}
$$

where $\alpha$ is the Maki parameter given by

$$
\alpha=\sqrt{2}\left[H_{\mathrm{c} 2}^{*}(0) / H_{\mathrm{p}}(0)\right]
$$

which later theoretical development has shown to be

$$
\alpha=2.35 \gamma \rho_{\mathrm{n}}=0.533\left[-\mathrm{d} \mu_{0} H_{\mathrm{c} 2} / \mathrm{d} T\right]_{T=T_{\mathrm{c}}} .
$$

Flux pinning. This property determines the critical current of the high field materials, a high value of $J_{\mathrm{c}}$ requires that the fluxoid lattice be held in place. Near the upper critical field the value of $J_{\mathrm{c}}$ is strongly affected by field. The formulation originally proposed by Fietz and Webb [16] with further development by Kramer [17] is most often used to express the dependence of the pin- ning force (defined as $J_{\mathrm{c}} B$ ) on applied field:

$$
F_{\mathrm{p}}=J_{\mathrm{c}} B=\mu_{0} H_{\mathrm{c} 2}^{n}\left(T^{\prime}\right) \mathrm{f}(h),
$$

where $h$ is the ratio of the applied field to the upper critical field and $n$ is an empirical constant that varies from $\sim 1.5$ to 2.5 . The function $\mathrm{f}(h)$ that describes the shape of the pinning curve is most often seen in the form $h^{p}(1-h)^{q}$ with the parameters $p$ and $q$ dependent on the specific materials.

Strain sensitivity. Practical superconducting materials, especially the intermetallics, are usually sensitive to strain. Their critical current and field are degraded by either compressive or tensile strain. Typical behavior of the measured critical field is shown in figure 8 . The horizontal axis is labeled intrinsic strain, illustrating an important point from the aspect of measurement; the superconducting material in a commercial wire may already be under strain (usually compressive) because of forces exerted by the stabilizer and other components of the composite. Thus, measurement of $H_{\mathrm{c} 2}$ on these practical materials becomes more of a problem than one might first imagine and leads to the definition of even more terminology. Most of this work comes under the heading of strain scaling studies and several empirical "laws" have emerged $[19,20]$ to describe the behavior. These scaling laws have the exact form of eq (12), but the temperature dependence of $H_{\mathrm{c} 2}$ is replaced by a strain dependence. Their application requires correct determination of the critical field even though, in general, they do not apply in either the low field $\left(<0.2 H_{\mathrm{c} 2}\right)$ or the high field $\left(>0.9 H_{\mathrm{c} 2}\right)$ regions. Two new defini-

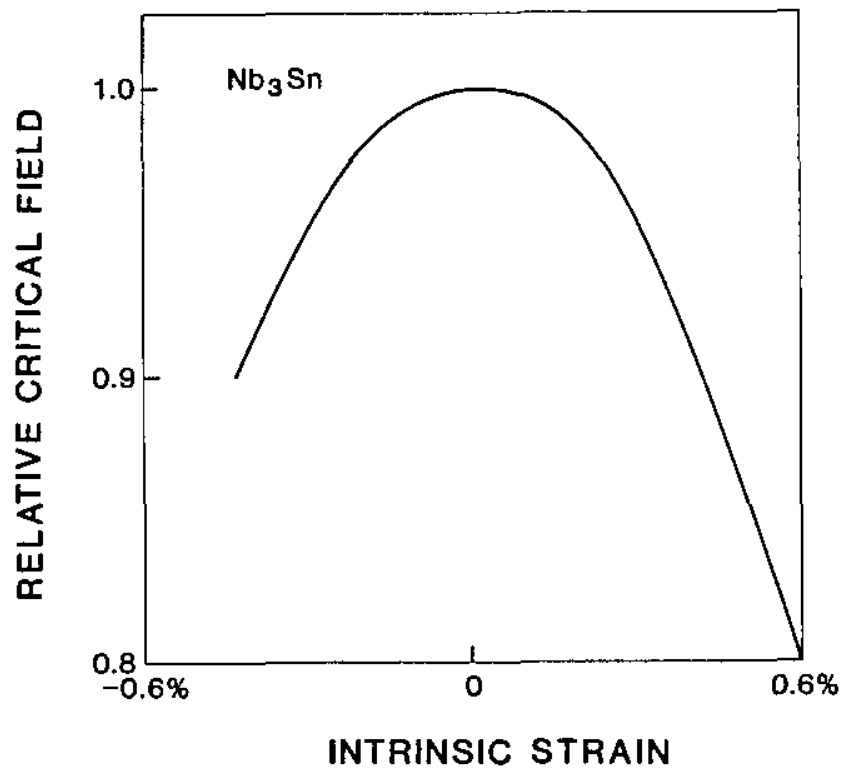

Figure 8-The effect of strain on the determination of the critical field. Data from [18]. 
tions appear in these writings: $H_{\mathrm{c} 20}$, which designates the "as received" value of $H_{\mathrm{c} 2}$ for a practical material and $H_{\mathrm{c} 2 \mathrm{~m}}$, which gives the maximum value of $H_{\mathrm{c} 2}$ under strain; the peak of the curve in figure 8 . This value is assumed to be that of the strain-free state of the superconductor. Since one is dealing with practical materials here, it should be pointed out that they do not often have a uniform composition or microstructure and $H_{\mathrm{c} 2}$ may well vary throughout the material. Thus, a sufficiently high measurement current is used so that a "bulk" value will be obtained (see the discussion of extrapolation techniques below) and this fact is often brought to the attention of the reader by use of $H_{\mathrm{c} 2}^{*}(\epsilon)$ as the critical field designation ( $\epsilon$ is the strain parameter).

\section{Measurement and Data Analysis Techniques}

Here we are concerned with measurement of the upper critical field, $H_{\mathrm{c} 2}$, although the techniques described could be applicable to the determination of other fields as well. The correct measurement of critical field, especially in practical superconductors, presents a difficult task both experimentally and in the interpretation of the resulting data. Different measurement methods may give different values for a given sample, and even the same method may give varying results depending on the choice of parameters, such as measuring current, used in a resistive determination. The cause of these variations is not an error in the concept or the measurement, but is most often due to material effects such as inhomogeneities in the superconductor. Thus, it sometimes happens that the measurement result that is most correct physically is not the most meaningful in a practical sense. Critical field determination, unlike the measurement of other superconductor parameters, suffers from the additional problem that one can seldom actu- ally achieve the fields necessary to see the transition directly and, thus, extrapolation techniques must be used which, themselves, are subjected to interpretation.

The literature on upper critical field measurements is mixed with regard to the care given in presenting the details of the experiment and the data analysis methods. Two authors have discussed aspects of the measurement problem in detail [21,22]. Many of their ideas and observations have been incorporated into the following sections.

\subsection{Defining the Transition}

Transitions between the superconducting and normal states are seldom as clean as the curves of figure 2 suggest. At best, the transitions have a width to them and at worst they are nearly lost in the noise. The techniques most commonly employed involve extrapolations of parts of curves as shown in figure 9. In practice, a more elaborate system is used as illustrated in figure 10, in which the "center" of the transition is taken as the midpoint between two points that may represent 25 and $75 \%$, as in the figure, or some other choice such as $10-90 \%$. This approach is helpful because the early parts of the transition may be obscured by noise and because of the unsymmetrical nature of the curve, but it is an arbitrary definition. In fact, it has been suggested that, for the resistive determination of critical field in practical materials, this technique is misleading, and the proper value should be taken as that at which the transition first appears with increasing field at a properly chosen current density [21]. This arrangement is used to avoid the problem of $H_{\mathrm{c} 2}$ variations throughout the superconducting material of the sample as discussed below. The author presents data showing that a significant difference in $H_{\mathrm{c} 2}$ value is found by the two techniques applied to commercial-type conductors. A similar comparison using single crystal Cu-Mo-S [23] found differ-
Figure 9-Techniques for determining critical field values from resistance and magnetization data plots [3].
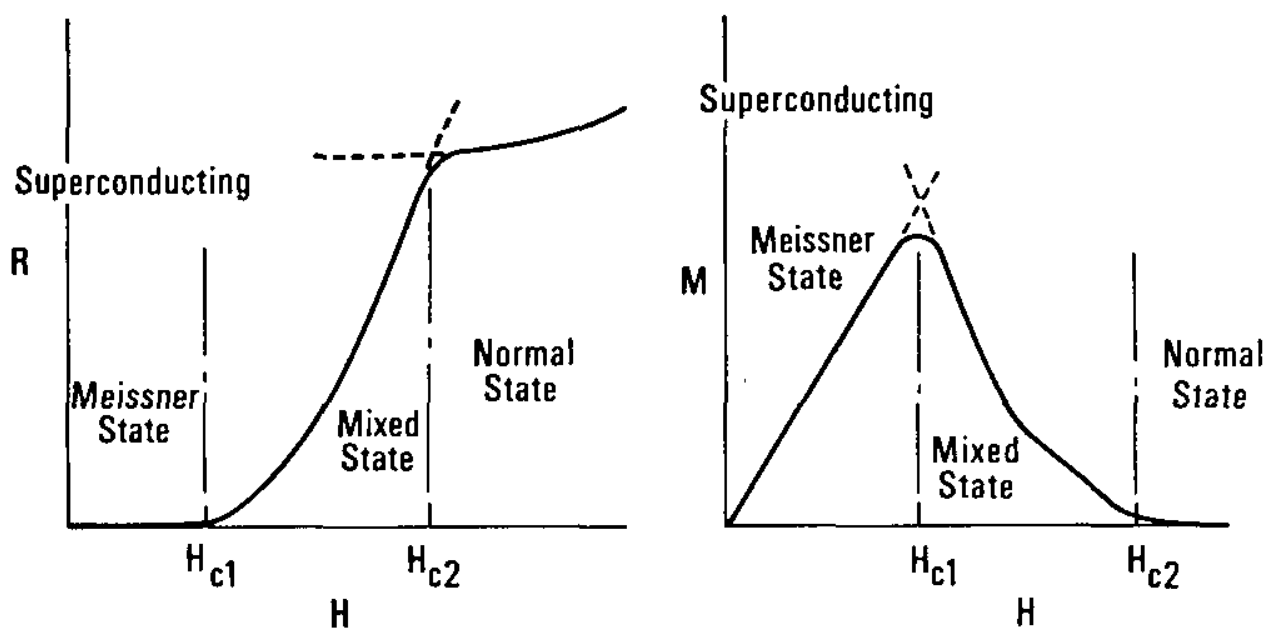


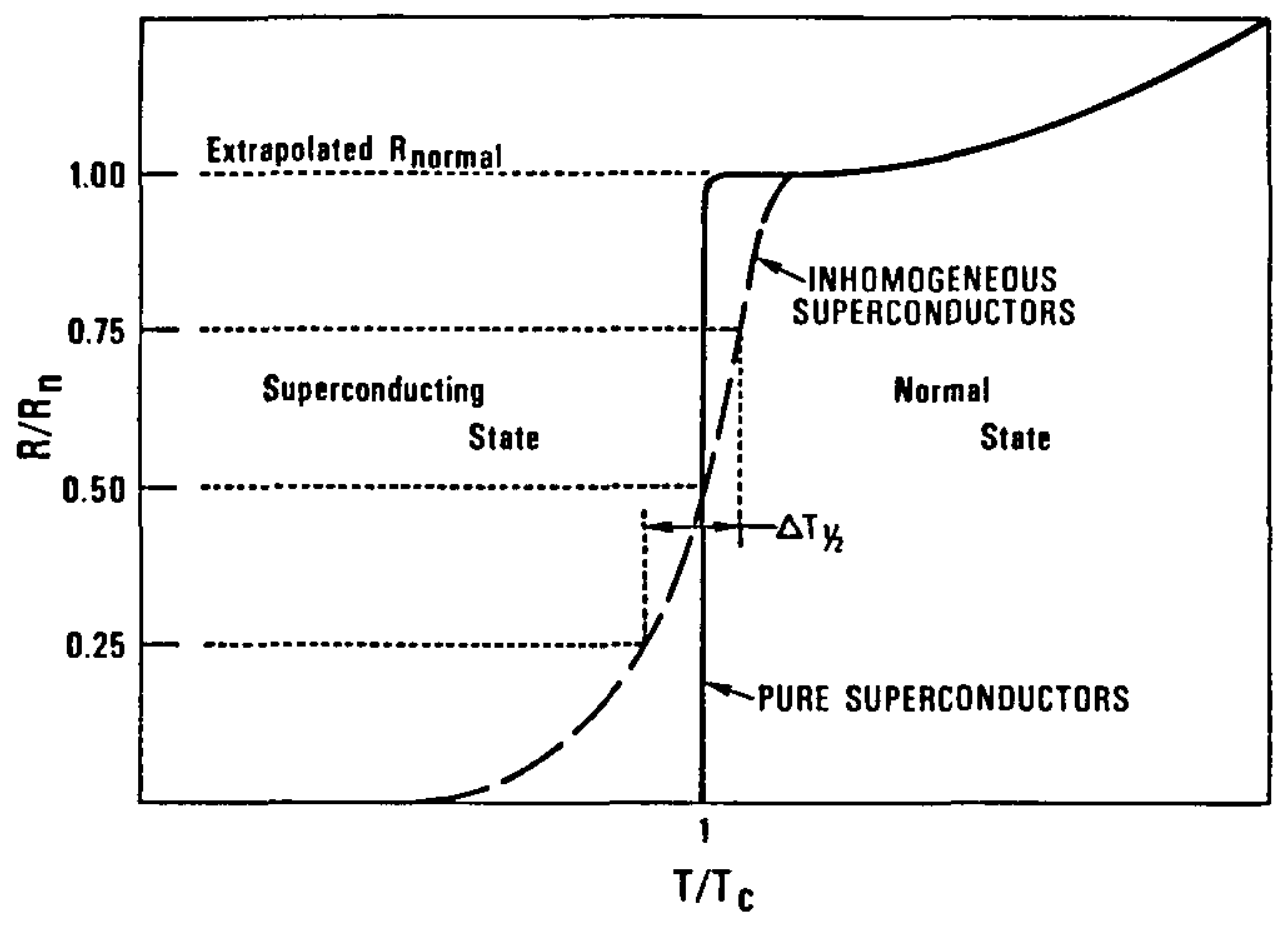

Figure 10-Illustration of method of determining the critical parameter value in the case of a broad transition [13].

ences of up to $20 \%$ between measurements using the two definitions.

A further problem can arise in the situation often encountered where one does not want to allow the sample to enter the normal state because of the possibility of damage. The approach in this case is to choose an arbitrary criterion of detected voltage over a fixed length of the wire as defining the critical parameter. This technique was chosen for the ASTM standard for critical current measurement [1]. The reasoning behind the choice and some of the related problems are discussed in an earlier publication [24]. The effect of the specific choice of criterion on the measured critical current is shown in figure 11 ; clearly a $1 \mu \mathrm{V}$ criterion results in a significantly lower critical current value than a $100 \mu \mathrm{V}$ criterion. As discussed in the reference, a low criterion is not always desirable in practical application because it may not be possible to measure it unambiguously. On the other hand, a very high one may put one too close to quenching the sample.

Figure 11-The effect of choice of criterion on the value reported for the critical current of a practical conductor. Numbers on the curves give the actual full-scale voltage in microvolts that corresponds to $100 \%$ on the vertical axis.

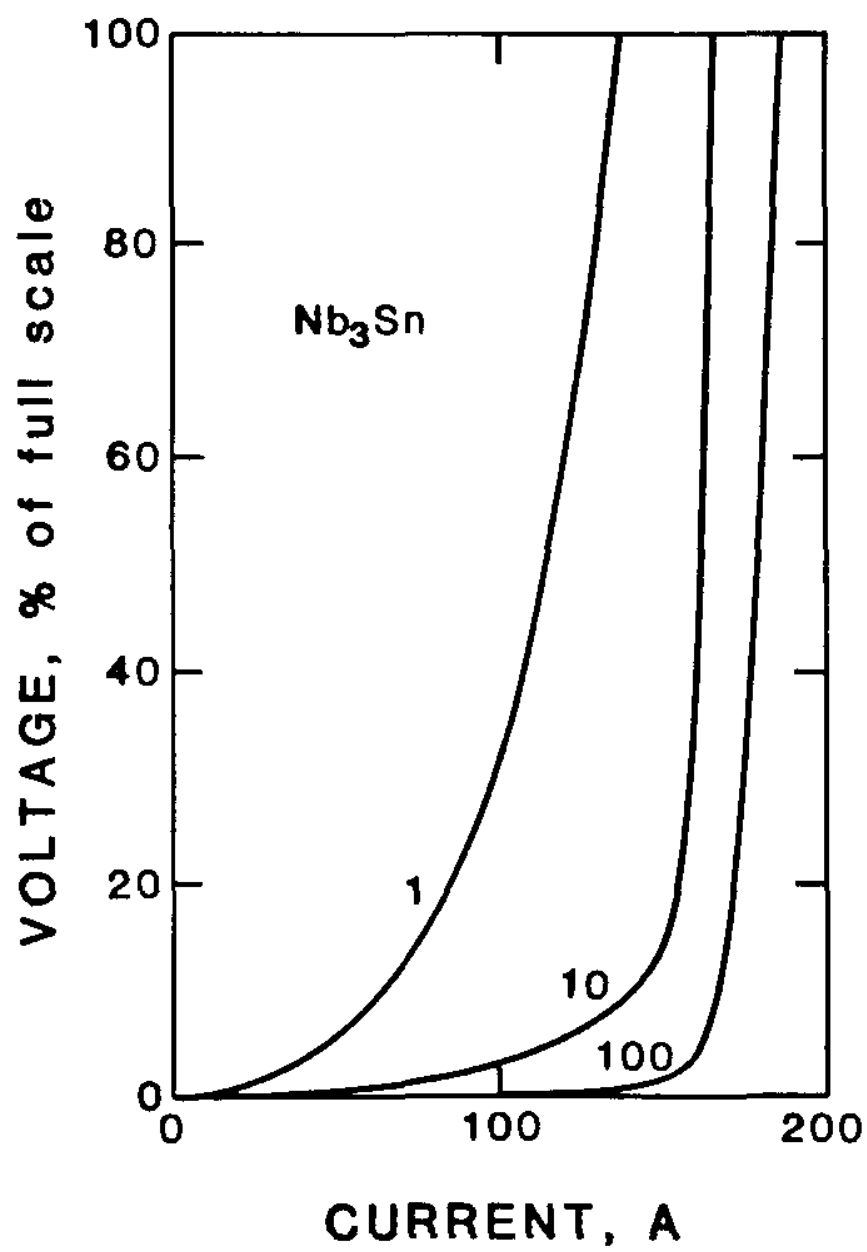


Because of its significance in understanding inhomogeneity effects, the width of the transition is an important feature of the data. All commercial materials show relatively broad transitions as indicated by the measurements shown in figure 12, especially those for the $\mathrm{Nb}_{3} \mathrm{Sn}$ samples. The interpretation is always strongly dependent on the measurement technique.

\subsection{Measurement Techniques}

A wide variety of measurement techniques has been used for the detection of the upper critical field of superconductors. The choice among them depends on the goal of the measurement and the time (and money) available. As always, the easiest measurements are the most difficult to interpret and the most subject to error-another manifestation of Murphy's Law. The techniques that have been used generally fall into five classes: electrical, magnetic, electromagnetic, thermal, and acoustic. For each of these we will discuss the theory behind the measurement, the ease of use, problems with the technique, and the data interpretation required. The apparatus for each method will be touched on briefly in the next section. We do not go into great detail here because a new book on materials properties measurement at low temperatures which thoroughly covers

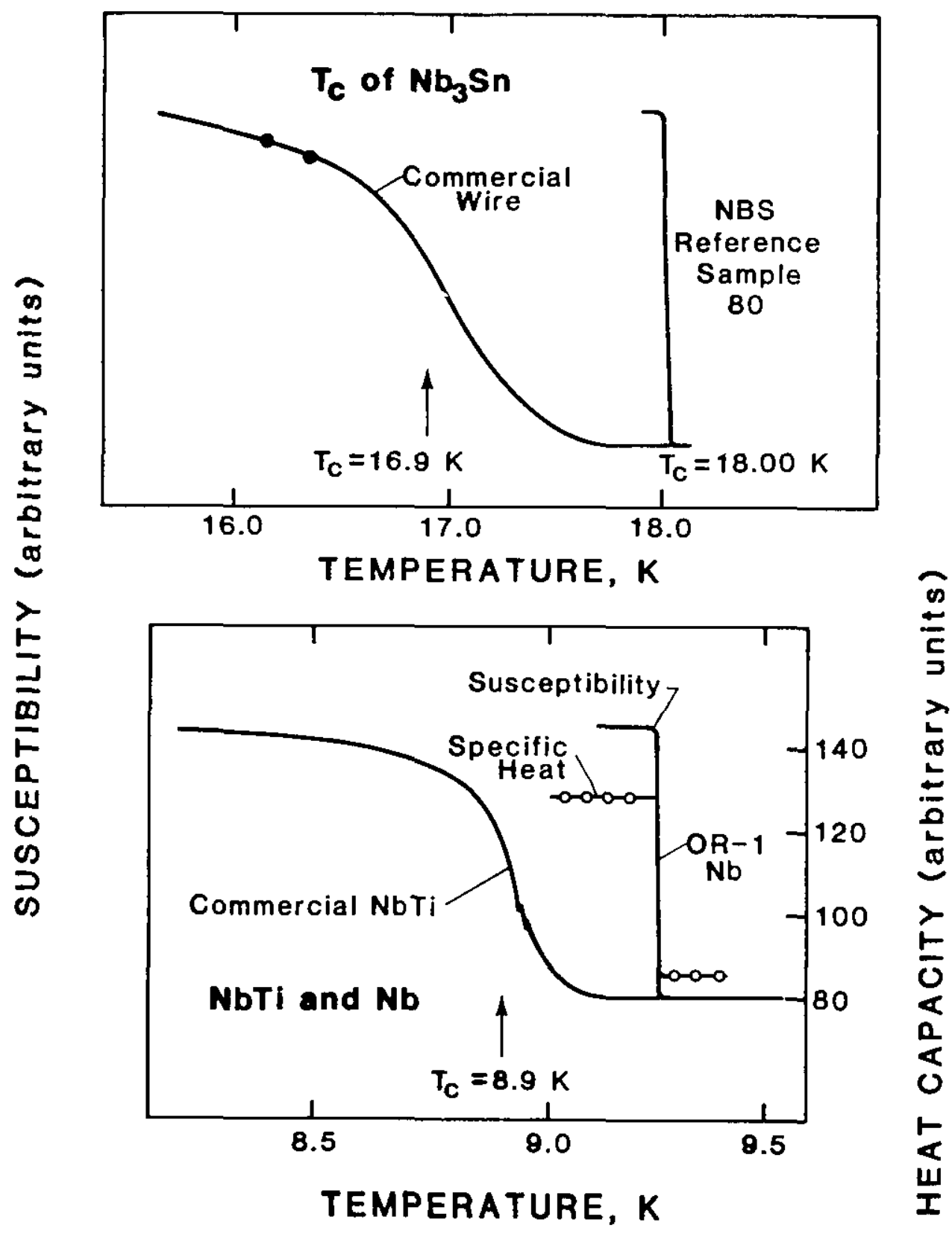

Figure 12-Comparisons between commercial materials and reference samples measured by several techniques [25]. 
each of the techniques, although not always their specific application to superconductors, has recently become available [26].

In the discussion that follows, one should keep in mind the fact that practical superconductors are inhomogeneous materials. The critical field of the superconductor may vary with the relative direction of the applied field and the conduction path. Isolated regions of superconducting material may occur, or regions may be coupled only through a percolation network or by the proximity effect. Normal conductor, usually copper, is almost always present, with a resistivity that is a factor of 100 or more below its room temperature value. Each of these factors will affect a given measurement method differently. The ideal measurement method would allow us to determine the microscopic variation of $H_{C_{2}}$ throughout the material, to measure the exact volume fraction of each superconducting species in the composite, and to determine the connectivity of the various regions. Thts would, of course, be accomplished with apparatus of minimum cost and greatest ease of operation. We may have to wait some time for this.

Resistive method. This measurement is by far the easiest to perform on commercial materials and it is the most commonly used. Care should be taken to have a long enough piece of wire that the minimum detectable voltage represents a reasonably low electric field criterion. Current transfer and current sharing effects can be minimized by having the voltage-measuring contacts on a significant length of the sample that is in a region of uniform field and distant from the current contacts by many wire diameters. This technique is probably the only common one that can reasonably be used in pulsedfield systems for the direct measurement of very high critical fields [27]. The values of $H_{\mathrm{c} 2}$ measured at low current levels can be high because the voltage will remain at zero with only a few strands still superconducting. In a few unusual situations (field parallel to surface, no normal metal jacket), it is also possible for surface superconductivity effects to cause the transition to be observed at very high field values. Even when surface effects are not of concern, the choice of measuring current is not a trivial problem. Larbalestier [21] presents data showing the value of $H_{\mathrm{c} 2}$ for cold-worked $\mathrm{NbTi}$ to vary from more than $11.6 \mathrm{~T}$ to less than $10.9 \mathrm{~T}$ as the measuring current density is raised from 0.005 to $10 \mathrm{~A} / \mathrm{cm}^{2}$, and the curve is not leveling out even then.

Magnetic methods. A number of techniques use detection of the change in magnetization of a sample as a means of monitoring the critical field transition. Early methods are described by Hein and Falge [28] and more recent ones in the book mentioned above [26]. At $H_{\mathrm{c} 2}$ both the magnetization and the susceptibility become effectively zero. Magnetometer techniques measure the magnetization of the sample directly. The sample is inserted into a pickup coil in the field region. The field is set to a fixed value and the sample is moved either out of the coil completely (ballistic method) or caused to oscillate within the coil (vibrating sample). The voltage induced in the coil is integrated to give the magnetization. A variation of this system is found in the swept-field technique in which the sample remains fixed within the pickup coil and the magnetic field is ramped at a fixed rate. Use of a balanced coil system (see below) allows extraction of the magnetization signal.

The various inductive techniques all use susceptometers; they measure the incremental susceptibility $(\Delta \mathrm{M} / \Delta \mathrm{H})$ by applying a small low-frequency ac field to the sample in the presence of a large background dc field and detecting the induced signal, either with a secondary coil (mutual inductance) or by the change in inductance of the primary (self inductance). The mutual inductance method is the most commonly used, and most often the secondary coil system is made up of two counterwound coils that are connected in series with the sample contained in one of them. This arrangement allows canceling of the primary field signal with the sample in the normal state prior to a measurement. Such systems are said to be balanced.

All of the systems just described require calibration if they are to be used for direct measurements rather than for tracking the sample through a transition. The easiest method uses a properly shaped test specimen of type I superconductor, such as lead, and assumes it is perfectly diamagnetic at $4 \mathrm{~K}$. Room temperature standards of susceptibility and magnetic moment are also available from the National Bureau of Standards. Magnetic methods are seldom actually used to determine $H_{\mathrm{c} 2}$ of practical materials, but they are used extensively in the determination of ac losses in superconductors. They are useful for samples of bulk material and for chips or powders. When used with wires, the samples are either bundles of cut wires with their axes parallel to the applied field or noninductively-wound coils in which the wire axis is normal to the field. Again, the inhomogeneous nature of practical superconducting materials can give problems in magnetic measurements. The most common occurs in superconductors where a high $-H_{\mathrm{c} 2}$ phase precipitates at the grain boundaries of lower critical field material. The magnetic techniques see this layer, and the resulting signal is indistinguishable from that which would be seen if the entire grain were actually in the superconducting state. In the "normal" situation of simply inhomogeneous superconductors, the inductive signal will tend to have a broader spread than the resistive one and, in general, will give a lower 
critical field value for a given material. Also, the magnetization change, $\Delta M$; tends to be very small for high$H_{\mathrm{c} 2}$ materials.

Specific heat. Measurement of the variation of the specific heat as a function of temperature through the superconducting transition is generally considered to be the best experimental method for characterizing superconducting materials of all sorts. It is the only one that can potentially indicate the amount of superconducting material present and, at least in concept, indicate the presence of superconductors with different transition temperatures or fields. However, it requires a relatively complex experimental apparatus for accurate measurements. Its use in the measurement of critical fields is further complicated by the effect of the field on the thermometry and the lengthy measurement times, but it has been used successfully for measurements on $\mathrm{Nb}_{3} \mathrm{Sn}$ in an $18 \mathrm{~T}$ field [29]. The temperature variation of the specific heat for an idealized superconductor is shown in figure 13. An excellent review of the theory and experiment with specific applications to superconductors is given by Stewart [30] and a general treatment of specific heat at low temperatures by Sparks [26]. Briefly, the discontinuity at the critical temperature arises from the different contributions to the specific heat made by normal and superconducting electrons. The lattice contribution is small at these temperatures; it varies as $T^{3}$ and is unaffected by the transition. The electronic contribution, on the other hand, is linear in $T$ in the normal state and takes an exponential form in the superconducting state with the exponent given by $-\Delta / \mathrm{k} T$, where $\Delta$ is the energy gap associated with the superconducting state. Furthermore, BCS theory gives the result

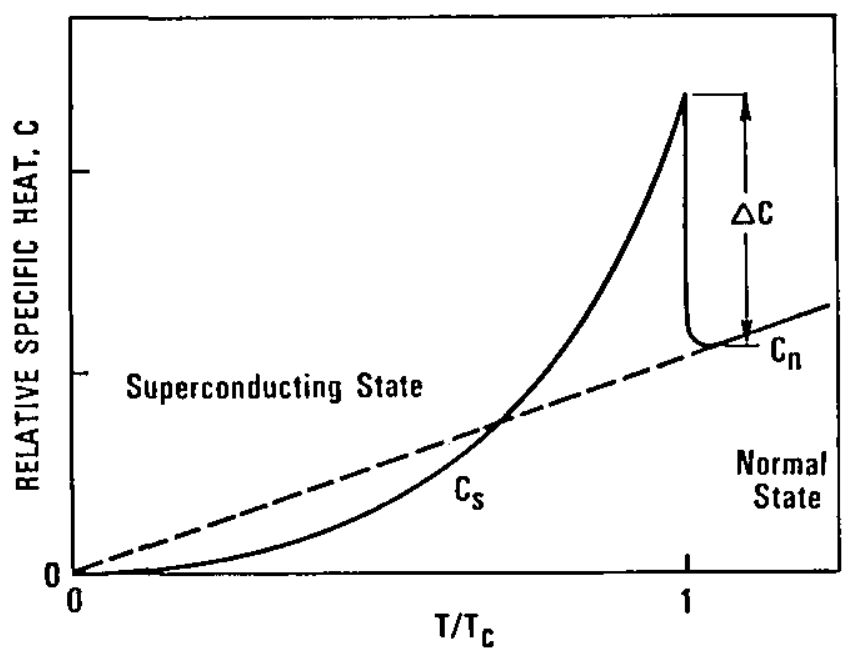

Figure 13-Behavior of the specific heat with temperature through a superconducting-to-normal transition [3].

$$
\mathrm{ae}^{-\Delta / \mathrm{k} T} \mathrm{c} / \gamma T_{\mathrm{c}}=2.43
$$

for the ratio of the specific heats of the superconducting and normal states at $T_{\mathrm{c}}$ in zero field. This allows one to estimate the amount of the sample that is superconducting, although the exact number may not hold true for some of the more exotic materials [30]. Furthermore, if data can be taken to well below the transition temperature, a much more accurate determination of the normal component of the sample can be made. Because of the temperature dependences just described, the only component of significance well below the transition is the $\gamma \mathrm{T}$ term of the normal material. The ratio of this term to the value of $\gamma \mathrm{T}$ above the transition is then a direct measure of the fraction of the sample that is not superconducting.

Other methods. The three techniques just mentioned are the only ones in common use for critical field determination. However, a few other methods have been used on occasion. Foner and colleagues [31,32] describe an $\operatorname{rf}\left(5\right.$ to $20 \mathrm{MHz}$ ) technique, useful for measuring $H_{\mathrm{c} 2}$ in powdered or odd-shaped samples, in which losses in an induced rf current are measured with a bridge arrangement. The technique is said to effectively monitor only the superconducting regions of the sample so that, in concept, it would allow determination of multiple critical field values of materials within a composite. The first of the papers also makes reference to a microwave technique.

The use of ultrasonics for critical field determination is discussed by Neuringer and Shapira [33]. The paper presents an in-depth analysis of the theory and experimental data showing the rapid increase in the attenuation of a $10 \mathrm{MHz}$ signal on field-induced transition to the normal state in a $\mathrm{NbZ} r$ alloy.

Comparisons. A few experiments have compared the critical temperature (not critical field) of a single sample as determined by several of the methods outlined above. They have (wisely) used samples of either pure type I material [34], carefully prepared niobium [25, see fig. 12], or single-phase alloys [33]. In all cases quite good agreement was obtained between measurements by all of the techniques, indicating that, in general, lack of agreement among different techniques applied to complex materials is due to the inhomogeneous nature of the composite rather than problems with technique. In fact, differences between the inductive and resistive methods have been used recently to investigate crack formation in the reaction layer of a $\mathrm{Nb}_{3} \mathrm{Sn}$ multifilamentary composite [35]. 


\subsection{Apparatus}

The specific apparatus required for the measurement of critical field by any of the techniques discussed here are adequately described in the references. General low temperature experimental methods are the subject of numerous texts (see [26] and references therein). Here we mention only two apparatus topics that are specific to critical field measurements, sample configuration and high-field magnet systems. For ease of discussion we assume that the sample is a wire and we use the resistive technique as our model, although the comments should usually apply to any other arrangement.

The measurement of critical parameters requires that the sample be mounted in such a manner that it will be as strain-free as possible after cooldown, but which assures that it will not be able to move under the effect of the applied field and current, while still maintaining good thermal contact with the bath. Figure 14 shows the most common sample mounting schemes. Each has its own pros and cons which have been discussed in detail elsewhere [24]. A particularly difficult problem has to do with the transfer of current in and out of the filaments in response to proximity to current contacts and to changes in field magnitude or orientation. Care must be taken that the measurement region is one in

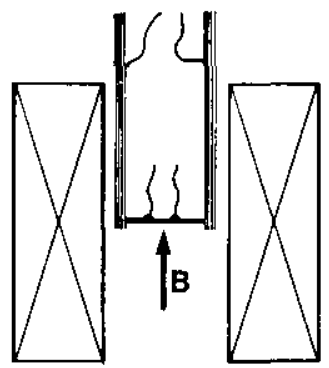

Short Straight

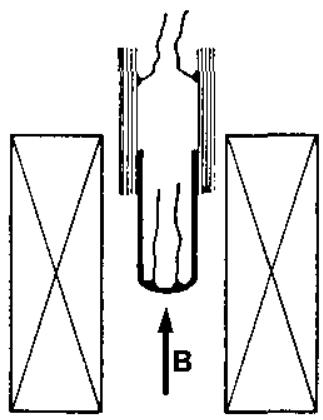

Hairpin
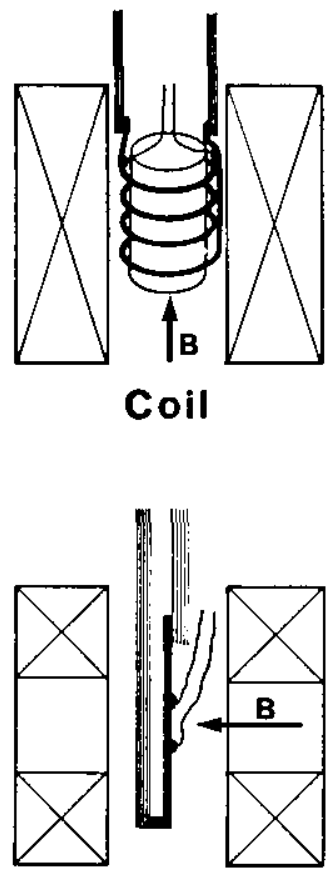

Long Straight
Figure 14-Commonly used configuration for samples for the measurement of critical parameters by the resistive method. which the current has attained a stable distribution. If the measurement involves sweeping a field, one should be aware that current transfer among filaments will be occurring during the sweep.

Magnet systems that attain fields much in excess of $10 \mathrm{~T}$ are not generally available. This makes direct measurement of the critical field of the more interesting materials at low current densities impossible. Thus, most laboratories use approaches that involve the extrapolation techniques discussed below. In the larger laboratories, steady fields of $12 \mathrm{~T}$ are available in reasonable volumes using superconducting magnets and fields to $20 \mathrm{~T}$ with normal magnets. Hybrid superconductingnormal systems exist that reach fields as high as $30 \mathrm{~T}$. Beyond that is the region attainable only with pulsed magnet techniques. These magnets allow one to achieve fields in the $50 \mathrm{~T}$ range for very short periods of time (tens of $\mathrm{ms}$ ) and the rapid field changes introduce a whole new set of problems into the measurement.

\subsection{Sources of Error}

It is possible to do a detailed error analysis for each of the critical field measurement methods described. However, it will nearly always show that the measurement itself can easily be made with an accuracy near $5 \%$ and somewhat better precision (near $2 \%$ ) if care is taken in the determination of the magnetic field and its uniformity and in the suppression of electronic noise. This level of accuracy is usually quite adequate for most purposes outside of the basic research laboratory. The real problem is the uncertainty in the critical field due to stress and nonuniformity of material in the sample and the effect of choices of criterion and measuring current or other experimental parameters on the final result, be it $H_{\mathrm{c} 2}$ or the transition width. Reasonable care in the preparation, characterization, and mounting of the samples is called for here. In pulsed field techniques care must be taken to prevent inductive heating of the material. Other rise-time effects appear to be negligible in the pulsed measurements [27]. In the more conventional measurements, slowly sweeping field or temperature seems to introduce no errors. At least it has been shown that the value and width of the transition is essentially the same whether one holds temperature fixed and sweeps the field or vice versa [36].

\subsection{Extrapolation Methods}

The value of the critical field for modern superconducting materials at the temperatures of interest is usually very high and, thus, unattainable in most laboratories. Several extrapolation methods are commonly used to allow determination of $H_{\mathrm{c} 2}$ at 0 or $4 \mathrm{~K}$ from other data on the critical parameters. The two common meth- 
ods are the use of data on the variation of $H_{\mathrm{c} 2}$ with $T$ near the critical temperature, where $H_{\mathrm{c} 2}$ is relatively low, and extrapolation using pinning theory of the critical current-versus-field behavior. Each of these methods depends critically on the use of theory to guide the extrapolation and each has reasonable success with certain materials and fails rather dramatically with others. For this reason, it is not possible to say that any one technique is "correct." It depends on the material to which it is applied. Also, these problems with correctness of the extrapolation are in addition to the problems already discussed related to the determination of the transition value from the experimental data. A small problem with terminology arises here also in that the extrapolated upper critical field is nearly universally designated $H_{\mathrm{c} 2}^{*}$, a term already used (see eq (7)) to indicate the theoretical critical field in the absence of paramagnetic limiting. In some cases there is no problem with this dual usage, but in others there definitely is the opportunity for some confusion.

Critical field versus temperature. This technique relies on the result from GLAG theory:

$$
H_{\mathrm{c} 2}^{*}(0)=a\left[\mathrm{~d} H_{\mathrm{c} 2} / \mathrm{d} T\right]_{T=T_{\mathrm{c}}} \cdot T_{\mathrm{c}},
$$

where $a$ is a constant equal to 0.69 for dirty materials and to 0.72 for clean ones. To use this method, the critical field value is measured in the region near $T_{\mathrm{c}}$ and the resulting plot is used to get the slope and the critical temperature. Typical data from the literature are shown in figure 15. The success of this method depends on how closely the material obeys the simple GLAG theory. The relationship in eq (14) can also be modified to account for various effects like paramagnetic limiting before the extrapolation is made, but this requires a knowledge of the material properties that may not exist. Also, the value of the differential is strongly dependent on the measuring current, with changes of as much as $30 \%$ reported for an order of magnitude change in the current [22]. As an example, note the widely different values obtained for the critical field of $\mathrm{Nb}_{3} \mathrm{Sn}$ in the two plots in figure 15 . One cannot really say that current density was the cause of the difference, but it is a strong possibility. Thus, the chance of agreement of an extrapolated measurement of this type with the upper critical field measured directly depends on the measurement parameters and also on the extent to which the subject material can be described by the various theoretical treatments available. In this latter situation, the common materials are in not too bad shape. Commercial quality $\mathrm{Nb}_{3} \mathrm{Sn}$ is agreed to be a simple GLAG-theory material, while the NbTi alloys show only a small amount of paramagnetic limiting of $H_{\mathrm{c} 2}$. On the other hand $\mathrm{V}_{3} \mathrm{Ga}$ has a strong paramagnetic limit and the more exotic materials, such as $\mathrm{Pb}-\mathrm{Mo}-\mathrm{S}$, cannot in general be fit to the theory even with the extensive modifications available for use in the literature.

There is another reason that one desires to have accurately measured data on the quantity $\mathrm{d} H_{\mathrm{c} 2} / \mathrm{d} T$ near $T_{\mathrm{c}}$ : it allows an experimental determination of the electronic specific heat coefficient, $\gamma$, that figures prominently in much of the theory. Comparison of eqs (15) and (5) show that the differential divided by the normal resistivity gives the value for gamma, assuming the proper theoretical value has been used for the proportionality coefficient. Application of this concept to numerous $\mathrm{NbTi}$ alloys is given by Hawksworth and Larbalestier [8].

Critical current versus field. Methods of assuring that a sample is at a fixed temperature other than that of a liquid helium bath are not easy to achieve experimentally. This fact makes the measurements just described quite difficult. An alternative technique is to use critical current data taken as a function of magnetic field at relatively modest fields and use pinning theory to extrapolate the data to zero critical current, which should occur at $H_{\mathrm{c} z}$. The success of this method depends not only on the correct measurement of the critical current, but also on knowledge of the exact behavior of the pinning force with field strength. For NbTi alloys, the expression,

$$
F_{\mathrm{p}}=\mathrm{c} H_{\mathrm{c} 2}^{2.5} \mathrm{~h}(1-\mathrm{h}),
$$

has proven useful in some instances [39], although the value of the exponent is not certain, being in the range 2.0 to 2.5 in most cases. Under the proper circumstances a simple linear extrapolation of $J_{\mathrm{c}}$ to zero is acceptable [22]. The material most investigated by this technique is probably $\mathrm{Nb}_{3} \mathrm{Sn}$. The pinning force expression as derived by Kramer (see the discussion for eq (12)) for this material is

$$
F_{\mathrm{F}}=K_{\mathrm{s}} \mathrm{h}^{\mathrm{t}}(1-\mathrm{h})^{2},
$$

where $K_{\mathrm{s}}$ is a constant for the conductor. The value of $K_{\mathrm{s}}$ and its dependence on $H_{\mathrm{c} 2}, \mathrm{k}$ and other parameters may change slightly depending on the particular theoretical modification chosen for the scaling [11]. In any event, it is conventional to rewrite eq $(10)$ as

$$
\left(J_{\mathrm{c}} H^{\mathrm{j}}\right)^{k}=K_{\mathrm{s}}^{\mathrm{i}} H_{\mathrm{c} 2}{ }^{-5 / 2}\left(H_{\mathrm{c} 2}-H\right),
$$

so that the equation becomes effectively linear in $H$. The quantity on the left hand side of the equation is then plotted as a function of applied field and a linear extrapolation made to zero. This method is illustrated by the 

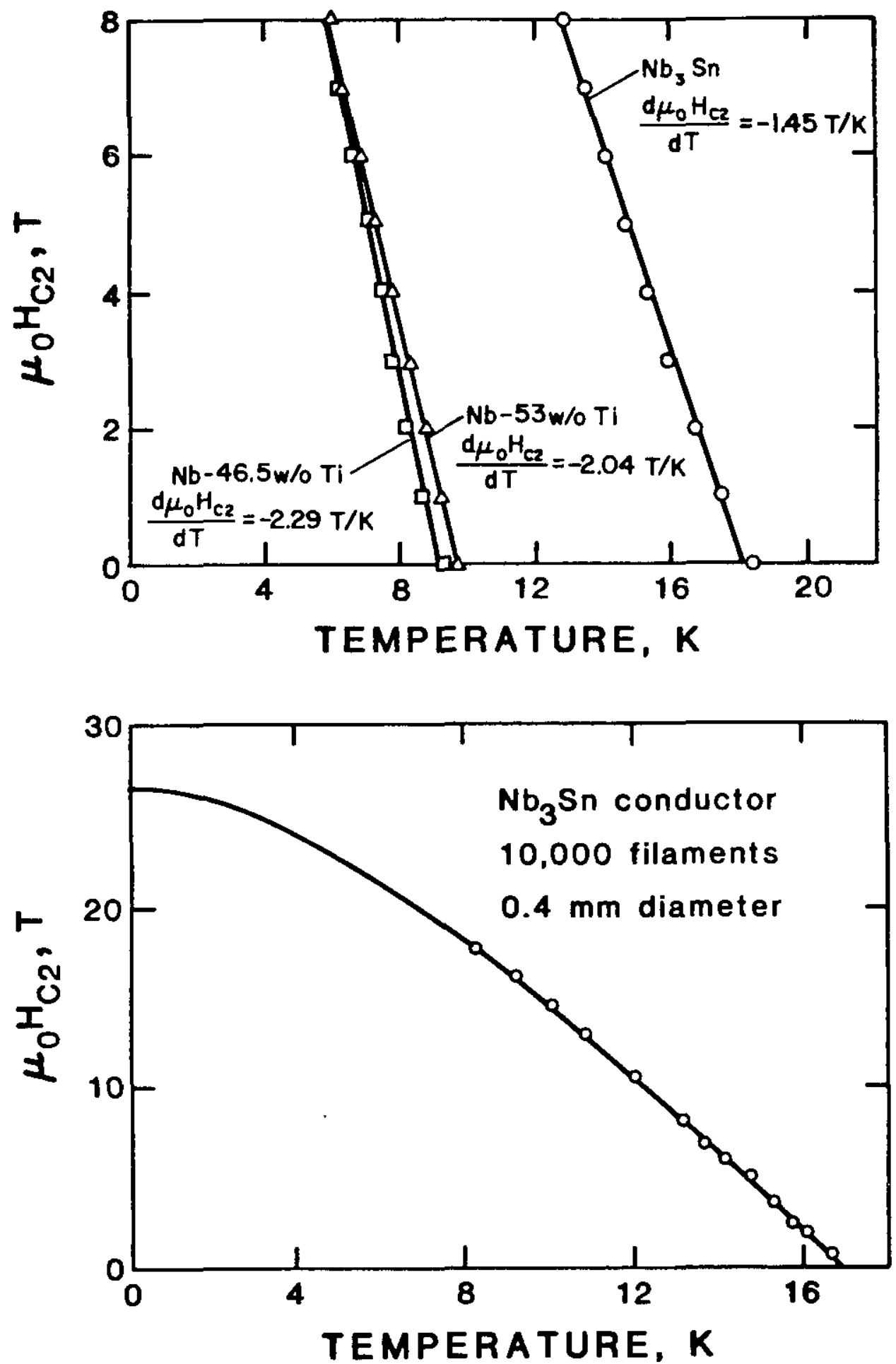

Figure 15-Typical data showing the behavior of the slope of $H_{\mathrm{c} 2}$ versus $T$ near $T_{\mathrm{c}}$ for several common materials. The upper graph is from [37] measured with a current density of $1.0 \mathrm{~A} / \mathrm{mm}^{2}$. The extrapolated value of $\mu_{0} H_{\mathrm{c} 2}$ for the $\mathrm{Nb}_{3} \mathrm{Sn}$ sample is $18.45 \mathrm{~T}$. The lower graph, from [38], shows data taken at a current density of $0.02 \mathrm{~A} / \mathrm{mm}^{2}$ and a fit to those data using the simple GLAG theory expression (eq (14)). plot of $4.2 \mathrm{~K}$ data shown in figure 16 in which the actual behavior of the curve is also shown, indicating the departure observed at low current values. This conductor is the same one as shown in the lower part cf figure 15 . The present extrapolation results in a significantly lower value for $H_{\mathrm{c} 2}$. In another instance the same author ob- served this method to give values for $\mathrm{Nb}_{3} \mathrm{Sn}$ that were too high compared to those obtained by direct measurement [20]. Furthermore, it has been seen that the extrapolation is not useful for highly aspected conductors such as tapes [11]. Application of the method to other high field materials requires a reevaluation of the 


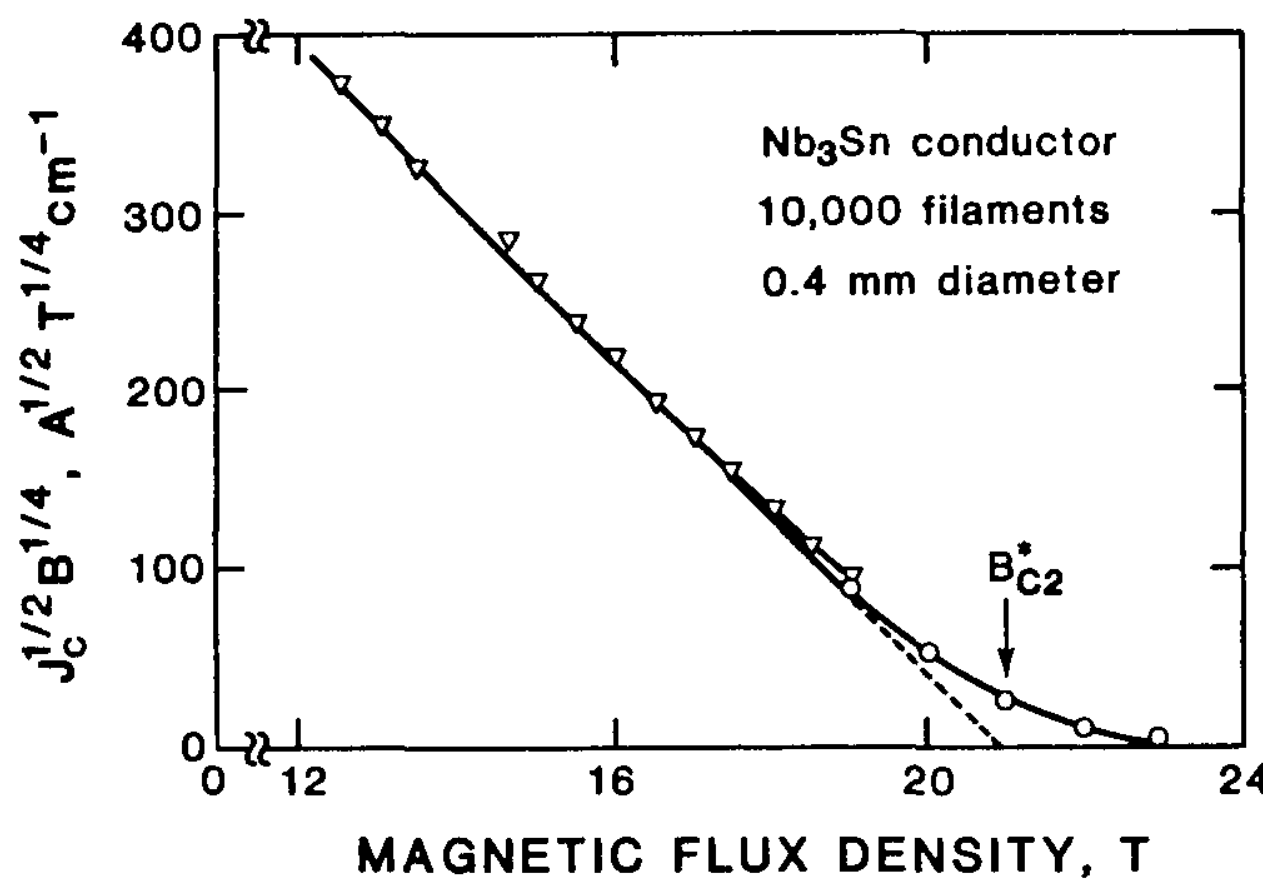

Figure 16-Determination of the upper critical field of $\mathrm{Nb}_{3} \mathrm{Sn}$ by the Kramer plot extrapolation [38]. The dashed line shows the extrapolation. Measured values are also shown in this region. Note that this sample is also the one for which data are shown in the lower part of figure 15.

constants used in eq (12) and effectively requires a more detailed analysis than the linear plot [19].

\subsection{Reporting of Data}

Since part of our goal is to evaluate the structure of a standard for critical field measurement, one consideration that is always of importance is the content of an adequate report of measurement. The many problems (or potential problems) outlined above seem to call for a very detailed report if an adequate assessment of the results is to be made by a reader interested in applying them to his own problem or evaluation. We suggest the following listing be used as a guide to the necessary components of a report. Clearly, not all parts will be required in every case.

\section{Results}

The critical field with correct symbol [e.g., $H_{\mathrm{c} 2}(T)$, $H_{\mathrm{c} 2}^{*}(0)$, etc.].

Measured directly or extrapolated.

Temperature of stated value (may or may not be temperature of measurement).

Estimated accuracy of the value given.

\section{Experiment}

Measurement technique used.
How the transition was determined from the raw data, including voltage or electric field criteria (where appropriate) and the point on the transition curve chosen for the reported value.

Width of the transition between stated limit points.

Details of sample geometry, internal structure, orientation with respect to the measuring field, mounting method considering questions of induced strain and thermal contact (for variable temperature methods).

Variable temperature methods require careful assessment of the effect of magnetic fields on the thermometry. Methods of calibration used to account for this effect should be described.

Each measurement method has its own set of reporting requirements. Most are the typical error and parameter choices related to any measurement with such a system. A few are of prime importance for critical field measurement reports.

Resistive transition-the current density, voltage probe separation, distance of voltage taps from the current contacts.

Magnetic technique-the exact method used, sample mass, magnitude of measurement fields (for inductive methods), calibration technique and accuracy, noise on unprocessed signal. 
Specific heat-thermometer calibration and relationship to sample temperature, sample mass, addenda loss evaluation, heater power levels.

Modern data collection techniques frequently involve processing of the data very early, perhaps even before it is seen by the experimenter. All such processing steps should be mentioned.

A point that is often neglected is the accuracy of the applied magnetic field measurement. Calibration of magnets, including evaluation of field inhomogeneity and, in the case of superconducting magnets, the effect of frozen-in flux is a difficult task. It is suggested that a calibration check be made every several months and certainly when the magnet system is first put into service.

\section{Extrapolation}

Details of an extrapolation technique should be given including:

The exact method used, including modifications to the theory employed and the actual equations.

The range of actual data used in the extrapolation.

If at all possible, a graph showing the data and the result of the extrapolation (see fig. 16).

\section{Conclusions}

As we mentioned at the beginning, the purpose of this investigation is to evaluate the desirability and feasibility of creating a standard for measurement of the critical field of practical superconducting materials. The type of standard uncer consideration is that typically produced by organizations such as ASTM to assist in commerce. Two standards already exist in the field of superconductivity, one for general definitions [4] and the other for the measurement of critical currents below $600 \mathrm{~A}$ [1]. Such standards are created by consensus among all interested parties and must be able to be used by industrial laboratories in their day-to-day operation. A further consideration is that there should be a demonstrated need for the standard, at least in the forseeable future.

A standard of this type can take several forms. It may be any of the following: a list of definitions; a manual outlining accepted measuring and reporting methods; a detailed method of measurement in which apparatus, technique, and report format are specified; or it may be an artifact or standard reference material. Whatever the form, it is essential that the standard be backed up with adequate research to document the need for each requirement of the standard. This is not a trivial problem, and it is often neglected in the rush to create a standard to solve a particular problem. Our feelings regarding the need for and structure of a standard are given below. In summary it seems that the time is not yet ripe for a full-fledged standard, but there is some justification for creating a list of standard definitions and, perhaps, an "operation manual" or similar document. A standard reference material approach might also prove useful, but would be quite expensive.

\subsection{Need for a Standard}

Standards of the sort discussed here are usually created in response to a need expressed by the community. In the case of critical field, there has been a limited expression of need. The commercial materials now in use are, in general, adequately characterized by their critical current versus field characteristic. Critical field information is of most use to that group of researchers who are trying to construct better practical materials for high field applications by modification of the crystallographic or electronic structure of various existing materials. This group should agree among themselves on the requirements for an acceptable measurement of the critical field, but that is not adequate reason for creating a standard. It is entirely conceivable that very high field materials may become feasible in the future, and the need could become great for a critical field standard for commercial versions of those superconductors. We do feel that a few definitions related to the critical field measurement should be added to the general definitions standard, mostly the various modifications of $H_{\mathrm{c} 2}$ discussed above. Furthermore, it is possible that certain groups, such as DoE, might want to specify a critical field measurement method and data analysis technique for a particular material. This could be done, but it would require that some of the research mentioned below be performed first if the document were to have very wide application.

\subsection{Measurement Standards}

As should be clear from the analysis above, the creation of a detailed single measurement standard for critical field is probably impossible given the current state of knowledge regarding the factors that influence $H_{\mathrm{c} 2}$ and the inhomogeneous nature of the superconducting portion of the practical conductor composite. However, if an attempt were to be made, there are a few items that should be consicered. The only measurement technique that is likely to be widely used in industry is the resistive method applied at $4.2 \mathrm{~K}$. A clever application of an inductive technique might also be possible, but none has 
appeared to date. Similarly, the possibility of routine direct measurement is remote because of the expense of high field magnets. Thus, extrapolation techniques would have to be used and most likely those would be critical current versus field extrapolations with the measurements made at $4.2 \mathrm{~K}$. Extensive research on the pinning force phenomenon would be necessary. Significant advances in understanding the effect of crystallographic and metallurgical variations on the critical field would be required. All these requirements could be mitigated somewhat by the use of a standard reference material as discussed below, but research on the measurement methods and their related errors would still be needed. A standard method should use a relatively high current density, probably in excess of $100 \mathrm{~A} / \mathrm{mm}^{2}$, which would avoid some of the problems, but would result in lower critical field values.

\subsection{Standard Reference Materials}

This approach to the standardization of critical field measurements is probably the most appealing for the present circumstances. Unfortunately, it is also a very expensive solution. The idea is to make a series of very well characterized materials that could then be distributed for the calibration of apparatus. Such materials could also be used to evaluate the various extrapolation techniques. The characterization would require very careful work, expertise in several measurement and analysis techniques, access to high field magnets, and a consensus as to the proper choices for the important parameters. However, considerable progress is now being made in understanding the interactions between the metallurgy and the superconducting properties of these materials which may well result in an advanced (practical) superconductor with well-documented homogeneity and internal structure in the near future. This conductor, if it can be made in significant quantities, would be an ideal candidate for a critical field SRM.

It is a pleasure to acknowledge the help of the entire NBS Superconductors and Magnetic Materials group, headed by A. F. Clark, through many conversations and suggestions. Special thanks are due R. B. Goldfarb for assistance with the subtleties of the field of magnetic units and J. W. Ekin for helping to keep the practicalities of the topic in the foreground. Much of the information on early experimental techniques was obtained from unpublished notes carefully prepared by D. T. Read. Mrs. V. Grulke cheerfully prepared the manuscript, with only occasional comments about amateur wordprocessor users.

\section{References}

Listed here are references from the recent literature in which the measurement or interpretation of critical field data are discussed. Those used as text references are numbered and make up the first part of the listing. The others, listed alphabetically following the text references, serve to provide background information. Only a few of the many excellent theoretical treatments are included.

[1] ASTM Standard for the Measurement of Critical Current (1983).

[2] Powell, R. L., and A. F. Clark, Definitions of terms for practical superconductors, 1. Fundamental states and flux phenomena, Crogenics 17, 697-701 (1977).

[3] Powell, R. L., and A. F. Clark, Definitions of terms for practical superconductors, 2. Critical parameters, Cryogenics 18, 137-141 (1978).

[4] ASTM Standard for Superconductor Terminology (1983).

[5] Matsumoto, H., and H. Unezawa, Interplay between magnetism and superconductivity, Cryogenics 23, 37-51 (1983).

[6] Fickett, F. R., and R. B. Goldfarb, Magnetic properties, Chapter 6 of Materials at Low Temperatures, American Society for Metals, Metals Park, Ohio (1983).

[7] Nelson, R. A., SI: The international system of units, published by the American Association of Physics Teachers, Stony Brook, NY (1983).

[8] Hawksworth, D. G., and D. C. Larbalestier, Enhanced values of $H_{\mathrm{c} 2}$ in $\mathrm{NbTi}$ ternary and quaternary alloys, Adv. Cryo. Eng. 26, 479-486 (1980).

[9] Cody, G. D., Phenomena and theory of superconductivity, Chapter 3 of Superconducting Magnet Systems, H. Brechna, ed., Springer-Verlag, New York, pp. 159-229 (1973).

[10] Beasley, M. R., New perspective on the physics of high-field superconductors, Adv. Cryo. Eng. 28, 345-359 (1982).

[11] Suenaga, M., and D. O. Welch, Flux pinning in bronzeprocessed $\mathrm{Nb}_{3} \mathrm{Sn}$ wires, in Filamentary A15 Superconductors, M. Suenaga and A. F. Clark, eds., Plenum Press, New York, pp. 131-142 (1980).

[12] Hulm, J. K., and B. T. Matthias, Overview of superconducting materials development, Chapter I of Superconducting Materials Science, S. Foner and B. B. Schwartz, eds., Plenum Press, New York, pp. 1-61 (1981).

[13] Ekin, J. W., Superconductors, Chapter 13 of Materials at Low Temperatures, American Society for Metals, Metals Park, Ohio (1983).

[14] Hulm, J. K.; J. E. Kunzler and B. T. Matthias, The road to superconducting materials, Physics Today, pp. 34-44 (Jan. 1981).

[15] Hulm, J. K., and B. T. Matthias, High-field, high-current superconductors, Science 208, 881-887 (1980).

[16] Fietz, W. A., and W. W. Webb, Hysteresis in superconducting alloys-temperature and field dependence of dislocation pinning in niobium alloys, Phys. Rev. 178, 657-667 (1969).

[17] Kramer, E. J., Scaling laws for flux pinning in hard superconductors, J. Appl. Phys. 44, 1360-1370 (1973).

[18] Ekin, J. W., Mechanical properties and strain effects in superconductors, Chapter 3 of Superconductor Materials Science, S. Foner and B. B. Schwartz, eds., Plenum Press, New York, 1981, pp. 455-509. 
[19] Ekin, J. W., Four-dimensional J-B-T- $\epsilon$ critical surface for superconductors, J. Appl. Phys. 54, 303-306 (1983).

[20] Rupp, G., Parameters affecting prestrain and $B_{\mathrm{c} 2}$ in multifilamentary $\mathrm{Nb}_{3} \mathrm{Sn}$ conductors, Adv. Cryo. Eng. 26, 522-529 (1980).

[21] Larbalestier, D. C., Superconducting materials-A review of recent advances and current problems in practical materials, IEEE Trans. Mag. MAG-17, 1668 1686 (1981).

[22] Evetts, J. E., The characterization of superconducting materials-Conflicts and correlations, IEEE Trans. Mag. MAG-19, 1109 (1983).

[23] Lee, Z. H., K. Noto, Y. Watanabe, and Y. Muto, A new anisotropy of the upper critical fields $H_{\mathrm{c} 2}$ in the $\mathrm{Cu}_{1.8} \mathrm{M}_{6} \mathrm{~S}_{8}$ single crystal, Physica 107B, 297-298 (1981).

[24] Goodrich, L. F., and F. R. Fickett, Critical current measurements: A compendium of experimental results, Cryogenics 22, 225-241 (1982).

[25] Schooley, J. F., and R. J. Soulen, Definition of the critical temperature of practical superconductors, in Development of Standards for Superconductors, F. R. Fickett and A. F. Clark, eds., NBSIR 80-1629 (1979) pp. 20-26.

[26] Materials at Low Temperatures, R. P. Reed and A. F. Clark, eds., American Society for Metals (1983).

[27] Fischer, O.; H. Jones, G. Bongi, M. Sergent, and R. Chevrel, Measurements of critical fields up to $500 \mathrm{kG}$ in the ternary molybdenum sulphides, J. Phys. C; Solid State Phys. 7, L450-L453 (1974).

[28] Hein, R. A., and R. L. Falge, Differential paramagnetic effect in superconductors, Phys. Rev. 123, 407-415 (1961).

[29] Stewart, G. R.; B. Cort and G. W. Webb, Specific heat of A15 $\mathrm{Nb}_{3} \mathrm{Sn}$ in fields to 18 Tesla, Phys. Rev. B 24, 3841 (1981).

[30] Stewart, G. R., Measurement of low-temperature specific heat, Rev. Sci. Instrum. 54, 1 (1983).

[31] Foner, S.; E. J. McNiff, Jr., B. T. Matthias, and E. Corenzwit, Properties of high superconducting transition temperature $\mathrm{Nb}_{3} \mathrm{Al}_{1-\mathrm{x}} \mathrm{Ge}_{\mathrm{x}}$ alloys, Proc. Eleventh International Conference on Low Temperature Physics II, 1025-11032 (eds. J. F. Allen, D. M. Finlayson, and D. M. McCall) St. Andrews (1968).

[32] Foner, S., High field superconductors, Colloq. Int'l. CNRS 242, 423-429 (1975).

[33] Neuringer, L. J., and $\mathrm{Y}$. Shapira, $\mathrm{Nb}-25 \% \mathrm{Zr}$ in strong magnetic fields: magnetic, resistive, ultrasonic, and thermal behavior, Phys. Rev. 148, 231-246 (1966).

[34] Soulen, R. J., and J. H. Colwell, Equivalence of the superconducting transition temperature of pure indium as determined by electrical resistance, magnetic susceptibility, and heat-capacity measurements, J. Low Temp. Phys. 5, 325-333 (1971).

[35] Cave, J. R., and C. A. F. Weir, Cracking and layer growth in $\mathrm{Nb}_{3} \mathrm{Sn}$ bronze route material, IEEE Trans. Mag. MAG-19, 1120 (1983).

[36] Habermeier, H.-U., Disorder and the upper critical field of $\mathrm{Nb}_{3} \mathrm{Ge}$, IEEE Trans. Mag. MAG-17, 1657-1659 (1981).

[37] Spencer, C. R.; P. A. Sanger and M. Young, The temperature and magnetic field dependence of superconducting critical current densities of multifilamentary $\mathrm{Nb}_{3} \mathrm{Sn}$ and $\mathrm{NbTi}$ composite wires, IEEE Trans. Mag. MAG-15, 76-79 (1979).

[38] Rupp, G.; E. J. McNiff, Jr. and S. Foner, Upper critical field in multifilamentary $\mathrm{Nb}_{3} \mathrm{Sn}$ conductors, IEEE Trans. Mag. MAG-17, 370-373 (1981).

[39] Hillmann, H., and K. J. Best, New measurements of critical data of optimized NbTi superconductors, IEEE Trans. Mag. MAG-13, 1568-1570 (1977).

\section{Critical Field Determination for Practical Superconductors}

Braginski, A. I., and H. C. Freygardt, Workshop on superconductors for magnets: Frontiers of technology, IEEE Trans. Mag. MAG-17, 2343-2354 (1981).

Bulakh, I. E.; S. V. Drozdova, C. A. Kalyushnaya, V. M. Pan, V. F. Primachenko, A. D. Shevchenko, N. V. Shevchuk, and M. V. Shubaev, Ultrahigh critical magnetic field superconductors $\mathrm{Pb}_{0.9} \mathrm{Mo}_{6} \mathrm{~S}_{7.5}$, IEEE Trans. Mag. MAG-17, 1642-1644 (1981).

Dew-Hughes, D., Practical superconducting materials, Chapter 2 of Superconducting Machines and Devices, S. Foner and B. B. Schwartz, eds., Plenum Press, New York, pp. 87-137 (1974).

Fietz, W. A., and C. H. Rosner, Advances in superconductive magnets and materials, IEEE Trans. Mag., pp. 239-259 (June 1974).

Flukiger, R.; S. Foner, E. J. McNiff, Jr., and B. B. Schwartz, Effects of material parameters on $H_{\mathrm{c} 2}$ in A15 superconductors, J. Mag. Mag. Mater. 11, 186-188 (1979).

Flukiger, R, Phase relationships, basic metallurgy, and superconducting properties of $\mathrm{Nb}_{3} \mathrm{Sn}$ and related compounds, Adv. Cryo. Eng. 28, 399-413 (1982).

Flukiger, R.; W. Schauer, W. Specking, L. Oddi, L. Pintschovius, W. Mullner, and B. Lachal, The phase relationships in $\mathrm{Nb}_{3} \mathrm{Sn}$ wires at low temperatures as detected by crystallographical (neutron and $x$-ray diffraction) and by physical $\left[B_{\mathrm{c} 2}(T), J_{c}\right.$ vs. $\left.\epsilon\right]$ measurements, Adv. Cryo. Eng. 28, 361-370 (1982).

Fujii, G., Present practices in Japan for the measurement and definition of various superconducting parameters, Cryogenics 21, 21-38 (1981).

Geballe, T. H., and J. K. Hulm, Superconductors in electric-power technology, Scientific American (November 1980).

Goncharov, I. N., and I. S. Khukharova, Investigation of the resistive behavior of a type-II superconductor near the upper critical field strength, Sov. Phys. JETP 35, 331-336 (1972).

Hoard, R. W.; R. M. Scanlan, G. S. Smith, and C. L. Farrell, The effect of strain on the martensitic phase transition in superconducting $\mathrm{Nb}_{3} \mathrm{Sn}$, IEEE Trans. Mag. MAG-17, 364-367 (1981).

Khan, H. R., and W. Schauer, Microstructure and superconductivity of some $\mathrm{C} 15$ laves phase alloys: $\mathrm{V}_{2} \mathrm{Hf}_{,} \mathrm{V}_{2} \mathrm{Hf}_{51} \mathrm{Nb}_{.49}$ and $\mathrm{V}_{2} \mathrm{Hf}_{.51} \mathrm{Ti}_{.28}$, IEEE Trans. Mag. MG-17, 1017-1020 (1981).

Landwehr, G., Problems connected with the generation of continuous magnetic fields above 15 Tesla, IEEE Trans. Mag. MAG-17, 1768-1774 (1981).

Larbalestier, D. C., Nb-Ti alloy superconductors-.-Present status and potential for improvement, Adv. Cryo. Eng. 26, $10-36$ (1980).

Larbalestier, D. C., Niobium-titanium superconducting materials, Chapter 3 of Superconductor Materials Science, S. Foner and B. B. Schwartz, eds., Plenum Press, New York, pp. 133-199 (1981).

Morohashi, S.; K. Noto, N. Kobayashi, and Y. Muto, Thermodynamic properties and fundamental parameters of single crystal $\mathrm{Cu}_{1.8} \mathrm{Mo}_{6} \mathrm{~S}_{8}$, Physica 1088, 929-930 (1981).

Orlando, T. P.; J. A. Alexander, S. J. Bending, J. Kwo, S. J. Poon, R. H. Hammond, M. R. Beasley, E. J. McNiff, Jr., and S. Foner, The role of disorder in maximizing the upper critical field in the $\mathrm{Nb}-\mathrm{Sn}$ system, IEEE Trans. Mag. MAG-17, 368-369 (1981).

Roberts, B. W., Survey of superconductive materials and critical evaluation of selected properties, J. Phys. Chem. Ref. Data 5, 581-821 (1976).

Scanlan, R. M., Superconducting materials, Añn. Rev. Mater. Sci. 10, 113-132 (1980).

Schauer, W., and W. Schelb, Improvement of $\mathrm{Nb}_{3} \mathrm{Sn}$ high field critical current by a two-stage reaction, IEEE Trans. Mag. MAG-17, 374-377 (1981)

Tachikawa, K., Recent developments in filamentary compound superconductors, Adv. Cryo. Eng. 28, 29-40 (1982). 